\title{
Deficiency of Mett|3 in Bladder Cancer Stem Cells inhibits Bladder Cancer Progression and Angiogenesis
}

\section{Ganping Wang}

Sun Yat-sen University First Affiliated Hospital

Yarong Dai

Shenzhen University

\section{Kang Li}

Sun Yat-sen University First Affiliated Hospital

\section{Maosheng Cheng}

Sun Yat-sen University First Affiliated Hospital

\section{Gan Xiong}

Sun Yat-Sen University Guanghua School of Stomatology

\section{Xiaochen Wang}

Sun Yat-sen University First Affiliated Hospital

\section{Shuang Chen}

Sun Yat-sen University First Affiliated Hospital

\section{Zhi Chen}

Sun Yat-sen University First Affiliated Hospital

Jianwen Chen

Sun Yat-sen University First Affiliated Hospital

\section{Xiuyun Xu}

Sun Yat-Sen University Guanghua School of Stomatology

\section{Liang Peng}

Chinese PLA General Hospital

Demeng Chen ( $\nabla$ chendm29@mail.sysu.edu.cn)

Sun Yat-sen University First Affiliated Hospital

\section{Research}

Keywords: Mett|3, cancer stem cell, bladder cancer, m6A, angiogenesis

Posted Date: November 18th, 2020

DOl: https://doi.org/10.21203/rs.3.rs-105980/v1 
License: (c) (i) This work is licensed under a Creative Commons Attribution 4.0 International License. Read Full License 


\section{Abstract}

Background: RNA N6-methyladenosine is a key step of post-transcriptional modulation which involves in governing gene expression. The $\mathrm{m}^{6} \mathrm{~A}$ modification catalyzed by Mettl3 has been widely recognized as a critical epigenetic regulation process for tumorigenic properties in various cancer cell lines, including bladder cancer. However, the in vivo function of Mettl3 in bladder cancer remains largely unknown.

Methods: Establishment of transgenic mouse model for exploring the role and mechanisms of Mettl3 in bladder cancer. Coupled global transcriptome sequencing and methylated RNA immunoprecipitation sequencing is performed to identify targets modulated by Mettl3.

Results: We found that ablation of Mettl3 in bladder urothelial attenuates the oncogenesis and tumor angiogenesis of bladder cancer. In addition, conditional knockout of Mettl3 in $\mathrm{K} 14^{+}$bladder cancer stem cell population leads to inhibition of bladder cancer progression. And deletion of Mettl3 leads to the suppression of TEK and VEGF-A through reduced abundance of $\mathrm{m}^{6} \mathrm{~A}$ peaks on specific region.

Conclusions: Taken together, Mettl3-mediated $\mathrm{m}^{6} \mathrm{~A}$ modification is required for the activation of TEKVEGF-A-mediated tumor progression and angiogenesis. Our findings may provide theoretical basis for bladder cancer treatment targeting Mettl3.

\section{Background}

Bladder cancer (BCa) is one of the most common malignancies worldwide, with approximately 200,000 death and 550,000 new cases yearly ${ }^{1}$. Patients with primary tumor could be treated by surgical resection, but it is limited by the several major progressions like metastasis and recurrence ${ }^{2,3}$. Furthermore, advanced $\mathrm{BCa}$ is characterized of high recurrence rate and poor prognosis, making it a growing social and medical challenge. During the past decades, despite great efforts on larger range of novel therapies for advanced $\mathrm{BCa}$, limited clinical efficacy is obtained ${ }^{4,5}$. Therefore, a deeper understanding of specific mechanism in BCa metastasis and recurrence as well as the exploration of effective targets for better disease control have become major goals to be attained.

N6-methyladenosine $\left(\mathrm{m}^{6} \mathrm{~A}\right)$, a predominantly internal modification of RNA in mammalian cells, is demonstrated to regulate RNA translation, degradation, splicing, export and folding ${ }^{6-9}$. And $\mathrm{m}^{6} \mathrm{~A}$ regulation is dynamic and reversible epigenetic modification mediated by three components: 'writer' complex, 'eraser' and 'reader'10-13. Methyltransferase-like 3 and 14 (Mettl3 and Mettl14) and their cofactors, Wilms tumor 1 associated protein (WTAP), VIRMA (KIAA1429) and RBM1 5 function as writers and form $\mathrm{m}^{6} \mathrm{~A}$ methyltransferase complex (MTC), which catalyzes $\mathrm{A}$ (adenosine) to become $\mathrm{m}^{6} \mathrm{~A}$. Emerging insights into the role of $\mathrm{m}^{6} \mathrm{~A}$ regulation in biological processes and pathogenesis of diseases has been uncovered in recent years, mainly including cell fate determination, embryonic development and tumor progression ${ }^{14,15}$. For example, data show $\mathrm{m}^{6} \mathrm{~A}$ modification pathway could exert carcinogenic or anticancer influence in various tumors for the expression pattern of the same $\mathrm{m}^{6} \mathrm{~A}$-regulated proteins and 
their target mRNAs may differ with the types of tumor ${ }^{16,17}$. Precious studies have demonstrated that Mett|3-mediated $\mathrm{m}^{6} \mathrm{~A}$ modification directly regulates AFF4/ NF-KB/MYC signaling network and ITGA6 $m R N A$, hence promoting $B C a$ progression ${ }^{18,19}$. However, the understanding of molecular mechanism of $\mathrm{m}^{6} \mathrm{~A}$ in $\mathrm{BCa}$ in vivo remains largely undefined.

Emerging evidences have shown that cancer stem cells can be used as a predictor for overall patient survival and process properties that are correlated with tumor progression, therapy resistance and recurrence ${ }^{20}$. CSCs in $\mathrm{BCa}$ are responsible for unresponsive chemotherapy and this unexpected consequence can be abrogated by celecoxib ${ }^{21}$. Once solid tumor grows up to a diameter of $2 \mathrm{~mm}$, vascular system is a necessity for continued growth. Then angiogenesis is acquired for tumor cells and function as a selective way to push the limits. Moreover, for tumor growth and invasion, angiogenesis has been considered to be the basic pathological feature during the process. TEK, also called TIE2, is an effective target for malignancy treatment by inhibition of tumor angiogenesis through ANG2/TIE2 axis ${ }^{22}$. VEGF-A is a well-known growth factor that activates angiogenesis to promote cell migration, therefore collaborating with tumorigenesis ${ }^{23}$. As a part of tumor comprehensive therapy, anti-angiogenesis therapy has been widely used in clinical practice. Furthermore, markers of angiogenesis CD31, CD34 and CD105 could severe as an independent prognostic factor in cancers ${ }^{24,25}$. Thus, understanding the specific pathway of regulating angiogenesis in $\mathrm{BCa}$ is essential for developing future diagnostic and therapeutic strategies.

In this study, we aim to identify the role that $\mathrm{m}^{6} \mathrm{~A}$ promotes $\mathrm{BCa}$ progression via focusing on the key methyltransferase Mettl3. Conditional knockout Mettl3 among K14 ${ }^{+}$or Upk3a ${ }^{+}$cells in vivo is found to restrain $\mathrm{BCa}$ development through disabling angiogenesis, which is further confirmed by RNA sequencing and analyzing the expression pattern of angiogenesis key factors. Collectively, we first verify the function of $\mathrm{m}^{6} \mathrm{~A}$ mediated by Mettl3 for $\mathrm{BCa}$ and expatiate on the network of Mett|3 in governing tumor angiogenesis in vivo.

\section{Methods}

\section{Animals}

The transgenic mice were maintained under specific pathogen-free conditions in Center of Experimental Animal of Sun Yat-sen University. All procedures and use of animal experiments were approved by Sun Yat-sen University Animal Care and Use Committee.

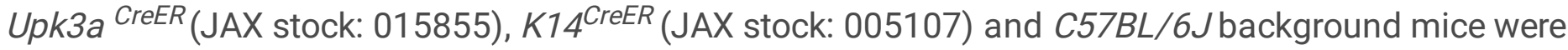
obtained from the Jackson Laboratory. Mett/3flox/flox and Mettl $3^{\mathrm{KI}}$ mice were obtained as previously described ${ }^{26}$. For induction of $\mathrm{BCa}$, six to eight weeks old mice were treated with drinking water containing $500 \mu \mathrm{g} / \mathrm{ml}$ BBN for 16 weeks and then given normal water for another 10 weeks. Tamoxifen was intraperitonelly injected to the mice with $0.08 \mathrm{mg} / \mathrm{g}$ of body weight each day for three days in order to 
inductively knock out the target gene. Then the experimental mice were sacrificed for collection of $\mathrm{BCa}$ samples.

\section{Immumohistochemical staining and IHC scoring}

All the BCa specimens collected were embedded in paraffin and then cut into $5 \mu \mathrm{m}$ thick sections. The samples were deparaffinized for 10 minutes in two different tanks with Histo-clear fluid after heating at $65^{\circ} \mathrm{C}$ for 30 minutes then these sections were hydrated in different gradients of ethanol. The samples were incubated for 10 minutes in $3 \% \mathrm{H}_{2} \mathrm{O}_{2}$ to block endogenous peroxidases. After blocking, antigen retrieval was performed with ethylenediaminetetraacetic acid (EDTA) buffer (Beyotime Biotechnology, Shanghai, China) in a microwave oven with medium power for 5 minutes and high power for another 5 minutes (avoid complete drying of sections). Specific primary antibody was used at $4^{\circ} \mathrm{C}$ overnight in a wet box. Then, anti-primary secondary antibody was applied and incubated for 30 minutes at room temperature. Removed the secondary antibody and dripped DAB upon sections, and observed the colour development index. Hematoxylin was conducted for counterstaining cell nucleus. The IHC score was calculated by the product of staining intensity and frequency. The intensity was defined as 0 (negative), $1+$ (weakly positive), $2+$ (positive) or $3+$ (strongly positive) and the frequency was considered to be percentage of positive cells.

\section{H\&E staining}

The sections were prepared by the same method as described above and stained with hematoxylin for 5 minutes. Next, hydrochloric acid alcohol was utilized to separate color for 5 seconds and the sections were further stained with eosin for 2 minutes. After that, all samples were dehydrated by gradient alcohol and sealed with neutral balsam medium.

\section{Immunofluorescence staining}

The BCa specimens were fixed in 4\% paraformaldehyde and permeabilized with $0.1 \%$ Triton X-100 (Sigma, US), followed by blocking with $1 \%$ bovine serum in phosphate-buffered saline (PBS) at room temperature. The sections were stained with primary antibody at 1:100 and were incubated at $4^{\circ} \mathrm{C}$ overnight, followed by application of the corresponding secondary antibody conjugated with DyLight 488 and Fluor 594 (Invitrogen, US). At the same time, normal serum was used as a control to guarantee the specificity of the primary antibody. The nuclei were counterstained with DAPI at 1:1000 for 1 minute. Images were captured with an upright fluorescence microscope (Nikon, Japan).

\section{RNA-sequencing}

Oligo (dT)-attached magnetic beads were used to purified mRNA from BBN-induced K14 ${ }^{\text {CreER }}$; Mett/ $3^{\text {flox/flox }}$ mice or their control littermates carrying bladder tumors. Purified mRNA was fragmented into small pieces with fragment buffer at appropriate temperature. Then First-strand cDNA was generated using random hexamer-primed reverse transcription, followed by a second-strand cDNA synthesis. Afterwards, A-Tailing Mix and RNA Index Adapters were added by incubating to end repair. The cDNA fragments obtained from previous step were amplified by PCR, and products were purified by Ampure XP 
Beads, and then dissolved in EB solution. The product was validated on the Agilent Technologies 2100 bioanalyzer for quality control. The double stranded PCR products from previous step were heated denatured and circularized by the splint oligo sequence to get the final library. The single strand circle DNA (ssCir DNA) was formatted as the final library. The final library was amplified with phi29 to make DNA nanoball (DNB) which had more than 300 copies of one molecular, DNBs were loaded into the patterned nanoarray and single end 50 bases reads were generated on BGIseq500 platform (BGIShenzhen, China).

\section{RNA extraction and RT-qPCR}

The bladder tissues were collected from BBN-induced mice carrying bladder primary tumor. Frozen tissues by liquid nitrogen were dissociated with $1 \mathrm{~mL}$ TRIzol Reagent (Invitrogen, US) per $50 \mathrm{mg}$ of tissue in Tissue Lyser. After dissociation, add $0.2 \mathrm{~mL}$ of chloroform per $1 \mathrm{~mL}$ of TRIzol Reagent and incubated for 3 minutes. Then, samples were centrifuged for 15 minutes at $12000 \times \mathrm{g}$ at $4^{\circ} \mathrm{C}$ and collected the upper aqueous phase containing total RNA. The supernatant was added equivalent isopropyl alcohol, gently blended and placed for $10 \mathrm{~min}$ at room temperature, followed by a centrifugation again and discard the supernatant. $1 \mathrm{~mL}$ of $75 \%$ ethanol diluted by DEPC water was added to each sample and the supernatant was discarded after the centrifugation $\left(4^{\circ} \mathrm{C}, 12000 \mathrm{r} / \mathrm{min}\right.$ and $\left.5 \mathrm{~min}\right)$. Moderate amount of DEPC-treated water was added to the precipitation after evaporation of ethanol. The $500 \mathrm{ng}$ of RNA from each sample was reverse-transcribed with the reverse-transcription kit (Takara Biotechnology Co., Ltd. Dalian, China). PCR reaction conditions were as follows: $98^{\circ} \mathrm{C}$ for 30 seconds, followed by 32 cycles at $95^{\circ} \mathrm{C}$ for 30 seconds, $55^{\circ} \mathrm{C}$ for 30 seconds, and $72{ }^{\circ} \mathrm{C}$ for 30 seconds. The specific primers for target genes in qPCR were as follows: TEK (forward), 5'- GAACTGAGGACGCTTCCACATTC- 3', TEK (reverse), 5'-

TCAGAAACGCCAACAGCACGGT- 3'; VEGF-A (forward), 5'- CTGCTGTAACGATGAAGCCCTG-3', VEGF-A (reverse), 5'- GCTGTAGGAAGCTCATCTCTCC- 3'; GAPDH (forward), 5'- AGGTCGGTGTGAACGGATTTG- 3', GAPDH (reverse), 5'- GGGGTCGTTGATGGCAACA- 3'.

\section{$\mathrm{m}^{6} \mathrm{~A}$-sequencing}

For $\mathrm{m}^{6} \mathrm{~A}$-seq, total RNA was isolated from bladder tissue with or without Mettl3 knockout using TRIzol reagent. mRNA was further purified from prepared total RNA with the use of the Dynabeads mRNA DIRECT kit (Thermo Fisher) according to the manufacturer's instructions. The purified RNA was used to interact with anti- $\mathrm{m}^{6} \mathrm{~A}$ or control rabbit IgG antibodies and the antibody-conjugated samples were incubated with IP buffer-washed Protein G Dynabeads at $4{ }^{\circ} \mathrm{C}$ overnight. Next day, the isolated products were applied for constructing libraries of inputs (control $\mathrm{lgG}$ ) and methylated RNA immunoprecipitation sequencing (meRIPs) (anti- $\mathrm{m}^{6} \mathrm{~A}$ antibody) with TruSeq Stranded mRNA Sample Prep Kits (Illumina RS122-2101). Libraries were multiplexed on an Illumina HiSeq 2000 platform. Mapping of reads, calling of m6A peaks, recognition of specific motifs and further analysis were carried out based on previous study ${ }^{27}$.

\section{KEGG and Gene Ontology Analysis}


To explore the link between Mettl3 and the underlying mechanisms in BCa, KEGG and Gene Ontology Analysis were conducted using ToppGene Suite (https://toppgene.cchmc.org/enrichment.jsp) and KEGG PATHWAY Database (https://www.kegg.jp/kegg/pathway.html) to analyze the DEGs. The statistical significance of difference was evaluated by $P$ value and $P<0.05$ was considered statistically significant.

\section{Gene Set Enrichment Analysis (GSEA)}

GSEA software downloaded from the GSEA website (https://www.gsea-msigdb.org/gsea/downloads.jsp) was performed to determine signaling pathways which were correlated between the Mettl3 knockout and control groups. The annotated gene sets of c5.go.v7.2.symbols.gmt in the Molecular Signatures Database (MSigDB) were selected in GSEA version 4.0. 1,000 times of permutations were performed. The normalized enrichment score (NES) was served as the GSEA main statistic. Gene sets with nominal $\mathrm{P}<$ 0.05, FDR $<0.25$ and NES $>1.0$ were considered significantly enriched.

\section{Statistical analysis}

All the data were analyzed and presented as mean \pm SD. Significant tests were conducted with unpaired parametric two-tailed Student's $T$ test for difference analysis between two groups or Chi-square for tumor grade analysis. $P<0.05$ was defined as statistical significance $(* P<0.05 ; * \star P<0.01 ; * \star \star P<0.001$; and $\star \star \star \star ~ P<0.0001)$. Data management and statistical analyses were performed using Graphpad Prism software, version 7.04 .

\section{Results}

\section{Deletion of Mett|3 in urothelium diminishes the development of mouse $\mathrm{BCa}$}

To explore the role of Mettl3 in BCa initiation and development, we first crossed Upk3aCreER mice with Mett/3flox/flox mice to generate Upk3aCreER mice; Mett/3flox/flox mice which allow us to conditional ablate the expression of Mettl3 in umbrella, intermediate, and basal cells of mouse bladder ${ }^{28}$. Upk3aCreER mice; Mett/3flox/flox and their control littermates were treated with BBN in their drinking water for 16 weeks before feeding normal water for another 10 weeks, as described previously ${ }^{29}$. Then mice were sacrificed for the collection of bladders. Measurement of bladder tumor indicated significantly decreased volume in Upk3aCreER, Mett/3 $3^{\text {flox/flox }}$ mice compared with the wild type group (Fig. 1A). We further assessed the effect of Mettl3 conditional knockout on tumor weight in vivo and found a marked tendency to reduce in tumor weight (Fig. 1B). Observation from the H\&E staining specimens demonstrated that mice developed lesions on the urothelium of the bladder upon the treatment with BBN while Upk3aCreER; Mett/ $3^{\text {flox/flox }}$ mice carried tumors were less malignant than in Upk3aCreER; Mett/3 ${ }^{w t / w t}$ mice (Fig. 1C). The result of BCa grading indicated a higher likelihood to develop into a high-grade tumor in wild type mice (Fig. 1D). IHC staining was applied to examine the protein levels of Mettl3, Ki67 and Caspase-3 protein in BCa (Fig. 1E). The reduced expression of Mettl3 and Ki67 with the increased level of Caspase-3 was detected by 
randomized double-blind IHC scoring in Upk3aCreER; Mett/3flox/flox mice, manifesting the role of Mettl3 in promoting proliferation and inhibiting apoptosis in vivo (Fig. 1F). These results indicate that Mettl3 is essential for cellular proliferation and survival in BBN-induced $\mathrm{BCa}$.

\section{Ablation of Mett|3 in $\mathrm{K} 14^{+}$cancer stem cells inhibits tumorigenicity and progression of $\mathrm{BCa}$}

We next investigated the function of Mettl3 in $\mathrm{K}_{14}{ }^{+} \mathrm{CSC}$ by generation of BBN-induced $\mathrm{K} 14^{\mathrm{CreER}}$, Mett/ $3^{\text {flox/flox }}$ and $K 14^{C r e E R}$, Mett/ $3^{w t / w t}$ mice. Bladder tumor size was obviously decreased after inducible knockout of Mettl3 in $\mathrm{K} 14^{+} \mathrm{CSC}$ (Fig. 2A). Similar to the results above, a notably reduced of tumor weight was observed with the knockout of Mettl3 (Fig. 2B). Then, BCa sections taken from transgenic mice were stained with H\&E kit to show the tumor histological morphology. The atypia of cells and tissues was more remarkable in wild type mice compared with $K 14^{C r e E R}$, Mett/ $3^{\text {flox/flox }}$ mice, revealing that

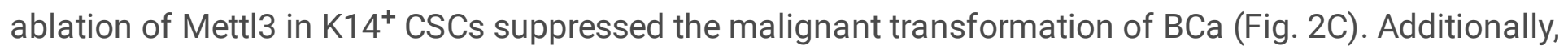
bladder tumor grading described Mettl3 as a driving factor of cancer progression (Fig. 2D). We then performed immunofluorescence staining to detect the expression K14 and expression level of Mettl3, Ki67 and Caspase-3 in our transgenic mice model. The data showed that Mettl3 was mostly knocked out after treatment with tamoxifen in K14-derived BCa cells followed by the suppression of Ki67 and enhancement of apoptosis, proving the effect of Mettl3 on elevating cell proliferative potential and limiting apoptosis (Fig. 2E, F). These data demonstrate that conditional knockout of Mettl3 in $\mathrm{K}_{14}{ }^{+} \mathrm{CSCs}$ reduced the tumor aggressiveness in mice with BBN induction.

\section{Overexpression of Mett|3 in urothelium or $\mathrm{K} 14^{+} \mathrm{CSCs} \mathrm{BCa}$ cells promotes the growth of $\mathrm{BCa}$}

Complementarily, we constructed mice models with inducible conditional over-expression of Mettl3 (Mettl $3^{\mathrm{KI}}$ ) in urothelium or $\mathrm{K}_{14}{ }^{+} \mathrm{CSC}$ using Upk3aCreER or $K 14^{\mathrm{CreER}}$. Comparison of the tumor bulk in Upk3a ${ }^{C r e E R}$ wild type and $\mathrm{KI}$ mice, the mice bearing BCa with overexpressed Mettl3 in Upk3a-dereved cells developed tumors more aggressively (Fig. 3A). It was also found that excessive Mettl3 in cells of Upk3a origin contributed to promoting tumor growth by measuring tumor mass in mice (Fig. 3B). Likewise, overexpression of Mettl3 in $\mathrm{K}_{14}{ }^{+} \mathrm{CSC}$ cells accelerated tumor growth and progression (Fig. 3C, D). IHC was performed to confirm the knock-in efficiency of Mettl3 (left) and there was an increased Ki67 positive cell along with enhanced staining intensity (right) after overexpression of Mettl3 (Fig. 3E). The level of Mettl 3 and Ki67 was found that was elevated in Upk3a ${ }^{C r e E R} \mathrm{KI}$ mice by estimating IHC score (Fig. 3F). Subsequently, Ki67 was also discovered to be upregulated in K14 ${ }^{C r e E R}$, Mettl3 knock-in mice (Fig. 3G, H). Taken together, our data demonstrate that over-expressed Mettl3 in bladder urothelium cells or $\mathrm{K}_{14}{ }^{+} \mathrm{CSCs}$ escalate the tumor aggressiveness in mouse BCa model. 


\section{Mett|3 regulates the PI3K/AKT pathway and tumor angiogenesis in $\mathrm{BCa}$}

To determine the downstream mechanism of how Mettl3 in regulating BCa progression, RNA-sequencing was conducted to investigate the mechanisms of Mettl3 in modulating downstream targets upon control and Upk3a/K14CreER; Mett/3 $c K O^{\text {flox/flox }}$ mice and differentially expressed genes were shown by separated into - down, -not significant (not sig.) and -up regulation with the use of Volcano scatterplot (Fig. 4A). Gene ontology of analysis in DEGs associated with biological process enrichment upon control and Mettl3 KO mice revealed that those genes were selectively enriched in regulation of angiogenesis, ameboidal-type cell migration, regulation of vasculature development and endothelial cell proliferation, which was likely involved in construction of tumor microenvironment and sustaining tumor metastasis (Fig. 4B). Next, we performed the KEGG enrichment analysis of down DEGs to systematically examine the cellular pathways affected by exhaustion of Mettl3 and PI3K-Akt signaling pathway was discovered to be the top enrichment of 13 pathways (Fig. 4C). In addition, some representative oncogenic features, such as PI3K signaling, epithelial-mesenchymal transition and angiogenesis were statistically enriched in Mettl3 conditional $\mathrm{KO}$ mice, elucidating that Mettl3 might regulate $\mathrm{BCa}$ in the aspect of oncogenesis, metastasis and tumor angiogenesis-mediated chemoresistance (Fig. 4D-F).

\section{Mett|3-dependent $\mathrm{m}^{6} \mathrm{~A}$ modification upon target mRNAs correlated with tumor angiogenesis and pathways in cancer}

To investigate whether Mettl3-mediated $\mathrm{m}^{6} \mathrm{~A}$ modification on direct targets could effectively influence biological process of BCa, we conducted methylated meRIP-seq to identify the role of Mettl3 in BCa. Firstly, the Pathway-Analysis displaying an obviously strong enrichment for pathways in cancer proved the great potential of Mettl3 in regulating BCa progression (Fig. 5A). The GO analysis on the gene set enriched by $\mathrm{m}^{6} \mathrm{~A}$ indicated Mettl3-medited methylation might participate in transcription regulation, protein phosphorylation and angiogenesis (Fig. 5B). We then applied Integrative Genomics View (IGV) software to search for the possible targets based on our meRIP-seq datasets and found that $\mathrm{m}^{6} \mathrm{~A}$ peaks were abundant upon TEK and VEGF-A genes that were responsible for tumor angiogenesis (Fig. $5 \mathrm{C}$ ). Consistent with the previous reports, our results showed the most enriched motif 'GGAC' in the specific regions of corresponding mRNAs (Fig. 5D).

\section{Mett|3 promotes bladder tumor angiogenesis via modulating TEK and VEGF-A}

For comprehensive understanding of the internal regulatory mechanisms that Mettl3 mediated bladder tumor angiogenesis, we utilized immunostaining to detect and localize the angiogenesis-related factors in vivo. Given that CD31 and CD34 were the primary indicators of endothelial cells and vascularassociated tissue, we explored their expression pattern in BCa sections by IHC. BCa with knockout of 
Mettl3 in urothelium exhibited apparently inhibition of angiogenesis (Fig. 6A, B). Similarly, the angiogenesis capacity was markedly declined among in $\mathrm{K} 14^{\mathrm{CreER}}$, Mett/3flox/flox $\mathrm{BCa}$ tissues (Fig. 6C, D).

We next quested whether loss of Mettl3 were able to inhibit the expression of specific targets involving in tumor angiogenesis as TEK and VEGF-A. IHC staining for assessment of downstream targets suggested that inducible knockout of Mettl3 in Upk3a-originated BCa cells significantly downregulated TEK and VEGF-A (Fig. 6E, F). Coincidently, these phenomena also could be observed in tumor sections collected from $K 14^{\text {CreER } W T}$ and KO mice (Fig. 6G, H). Concordant results were found performing RT-qPCR for detecting the mRNA level of TEK and CD34. Relative mRNA expression of TEK and VEGF-A among the Upk3aCreER, Mettl3 KO mice exerted dramatically reduced versus Upk3aCreER, Mett/3 ${ }^{\text {wt } / \text { wt }}$ (Fig. 6I). Furthermore, ablation of Mettl3 in K14-derived BCa cells dramatically decreased VEGF-A level (Fig. 6J). Collectively, these data has testified that Mettl3 exerted its effect on tumor angiogenesis, supporting the role of Mettl3 as a collaborator of BCa progression by modulation of neovascularization.

\section{Discussion}

Recently, RNA $\mathrm{m}^{6} \mathrm{~A}$ methyltransferase Mettl3 has been reported to modulate AFF4/NF-KB/MYC signaling for $\mathrm{BCa}$ progression in vitro ${ }^{18}$. However, the role of Mettl3 in BCa progression and tumor microenvironment regulation in vivo remains elusive. In this study, we show that Mettl3 facilitates $\mathrm{BCa}$ progression by mediating tumor angiogenesis in vivo using transgenic mouse model. Conditional depletion of Mettl3 among urothelium or $\mathrm{K} 4^{+}$CSCs significantly restricts oncogenesis and prevents $\mathrm{BBN}$-induced $\mathrm{BCa}$ from progressing to more aggressive stage. In addition, Mett|3 ablation leads to downregulation of proliferative index protein $\mathrm{Ki} 67$ and upregulation of apoptosis-related Caspase-3, indicating a role of Mettl 3 in cell proliferation and survival of $\mathrm{BCa}$. The global transcriptome sequencing with KEGG Analysis reveals that Mettl3 is closely related to tumor angiogenesis. Furthermore data generated from $\mathrm{m}^{6} \mathrm{~A}$-squencing portray a regulatory network of Mett|3-modulated TEK/PI3K/VEGF axis which propels the angiogenesis surrounding tumor cells. Indeed, the IHC staining results blood vessels markers, CD31 and CD34, confirmed that the absence of Mettl3 in urothelium or K14 ${ }^{+}$CSCs reduces the level of angiogenesis in $\mathrm{BCa}$ tissues. Our findings offer convincing evidences of Mettl3 in epigenetically modifying TEK/PI3K/VEGF cascades involving in angiogenesis, suggesting that therapeutically targeting Mett|3 may provide one of the alternatives for anti-angiogenesis therapy in bladder carcinoma.

The $\mathrm{m}^{6} \mathrm{~A}$ modification catalyzed by Mettl3 has been proven to control specific target mRNAs translation efficiency and stability, which plays a key role in promoting progression of various cancers including lung cancer, endometrial cancer, leukemia and colorectal cancer ${ }^{30-33}$. Mechanically, Mettl3 could activate ZMYM1 (zinc finger MYM-type containing 1) towards genes complex of chromatin via $\mathrm{m}^{6} \mathrm{~A}$ modification, hence promoting EMT (epithelial-mesenchymal transition) and metastasis of gastric cancer ${ }^{34}$. Herein, we report that the mRNAs enriched by $\mathrm{m}^{6} \mathrm{~A}$ peaks are downregulated by loss of Mettl3 likely as a result of mRNA instability. However, whether the extinction of Mettl3 interferes translation efficiency of these targets is still unclear, which calls for further research. 
Insights of regulatory mechanisms in tumor angiogenesis expand our horizons of the crosstalk between tumor cells and surrounding microenvironment. Previous study has shown Ang2-TEK signaling as key regulator in lymphogenous metastasis ${ }^{35}$. VEGF-A as a downstream factor of Zeb1 enhances breast cancer cells aggressiveness through sustaining stemness. Indeed, the regulatory networks of epigenomics, angiogenesis and stemness maintenance are intricate. A recent study describes a more faithful murine BCa model that carries Upk3a-Cre ${ }^{\text {ERT2; }}$ Trp53 ${ }^{\mathrm{L} / \mathrm{L}}$; Pten ${ }^{\mathrm{L} / \mathrm{L}}$; Rosa26 $6^{\mathrm{LSL}-\mathrm{Luc}}$ tumor is applied to excavate biology of $\mathrm{BCa}^{36}$. Another research indicates that the subpopulation of bladder basal cells characterized by K14 possesses self-renewal ability and these cells are defined as the origin of urothelial cancer, which contributes to BCa progression ${ }^{37}$. Encouragingly, combination of these rationales of signal networks in BCa may depict the extensive interactions of stemness maintenance, epigenetic modulation and tumor angiogenesis. Our results have revealed a novel mechanism of Mettl3 by which mediates modulation of TEK and VEGF-A to promote tumor angiogenesis.

In summary, out data demonstrate the critical role of Mett|3 in driving BCa progression, as featured by expediting angiogenesis surrounding tumor cells, and unveil a previously unrecognized signaling axis involving Mett|3-TEK-VEGF-A-CD31/CD34 in bladder malignancy. More importantly, our studies propose that targeting Mettl3 might bring profound influence on conquering chemotherapy resistance and immunotherapy tolerance caused by tumor angiogenesis.

\section{Conclusions}

Our findings expound a novel molecular mechanism which regulates the TEK-VEGF-A-CD31/CD34 pathway and angiogenesis in BCa by Mettl3 using transgenic mouse model.

\section{Abbreviations}

$\mathrm{BCa}$

bladder cancer

$\mathrm{m}^{6} \mathrm{~A}$

N6-methyladenosine

Mettl3

Methyltransferase-like 3

WTAP

Wilms tumor 1 associated protein

MTC

methyltransferase complex

EDTA

ethylenediaminetetraacetic acid

PBS

phosphate-buffered saline 
ssCir DNA

single strand circle DNA

DNB

DNA nanoball

meRIP-seq

methylated RNA immunoprecipitation sequencing

GSEA

Gene Set Enrichment Analysis

MSigDB

Molecular Signatures Database

NES

normalized enrichment score

IGV

Integrative Genomics View

ZMYM1

zinc finger MYM-type containing 1

EMT

epithelial-mesenchymal transition

\section{Declarations}

Ethical Approval and Consent to participate: The authors declare no conflict of interests in this work. All institutional and national guidelines for the care and use of laboratory animals were followed.

Consent for publication: Written informed consent for publication was obtained from all participants.

Availability of supporting data: The data sets supporting the results of this article are included within the article and its additional files.

Competing interests: The authors declare no conflict of interests in this work.

Funding: This paper was supported by grants from the Natural Science Foundation of Guangdong Province (no. 1814050001877) and the National Natural Science Foundation of China (no. 8187111008).

Author contributions: Demeng Chen and Liang Peng conceived and were in charge of this project. Ganping Wang, Yarong Dai, Kang Li, Maosheng Cheng, Gan Xiong, Xiaochen Wang, Shuang Chen, Zhi Chen, Jianwen Chen and Xiuyun Xu conducted experiments under the supervision of Demeng Chen. Kang Li perform bioinformatics analysis under the supervision of Demeng Chen and Liang Peng. Ganping Wang and Demeng Chen wrote the manuscript.

Acknowledgements: Not applicable. 


\section{References}

1. Bray F, et al. Global cancer statistics 2018: GLOBOCAN estimates of incidence and mortality worldwide for 36 cancers in 185 countries. Cancer J Clin. 2018;68:394-424.

2. Griffiths TR. \& Action on Bladder, C. Current perspectives in bladder cancer management. Int J Clin Pract. 2013;67:435-48.

3. Crabb SJ, Douglas J. The latest treatment options for bladder cancer. British medical bulletin. 2018;128:85-95.

4. Powles T, et al. Efficacy and Safety of Durvalumab in Locally Advanced or Metastatic Urothelial Carcinoma: Updated Results From a Phase 1/2 Open-label Study. JAMA oncology. 2017;3:e172411.

5. Powles T, Morrison L. Biomarker challenges for immune checkpoint inhibitors in urothelial carcinoma. Nature reviews Urology. 2018;15:585-7.

6. Wang X, et al. N(6)-methyladenosine Modulates Messenger RNA Translation Efficiency. Cell. 2015;161:1388-99.

7. Wang X, et al. N6-methyladenosine-dependent regulation of messenger RNA stability. Nature. 2014;505:117-20.

8. Zhao X, et al. FTO-dependent demethylation of N6-methyladenosine regulates mRNA splicing and is required for adipogenesis. Cell research. 2014;24:1403-19.

9. He L, et al. Functions of N6-methyladenosine and its role in cancer. Mol Cancer. 2019;18:176.

10. Liu J, et al. A METTL3-METTL14 complex mediates mammalian nuclear RNA N6-adenosine methylation. Nature chemical biology. 2014;10:93-5.

11. Jia G, et al. N6-methyladenosine in nuclear RNA is a major substrate of the obesity-associated FTO. Nature chemical biology. 2011;7:885-7.

12. Zheng G, et al. ALKBH5 is a mammalian RNA demethylase that impacts RNA metabolism and mouse fertility. Molecular cell. 2013;49:18-29.

13. Li A, et al. Cytoplasmic m(6)A reader YTHDF3 promotes mRNA translation. Cell research. 2017;27:444-7.

14. Roundtree IA, Evans ME, Pan T, He C. Dynamic RNA Modifications in Gene Expression Regulation. Cell. 2017;169:1187-200.

15. Frye $\mathrm{M}$, Harada BT, Behm M, He C. RNA modifications modulate gene expression during development. Science. 2018;361:1346-9.

16. Wang S, Chai P, Jia R, Jia R. Novel insights on m(6)A RNA methylation in tumorigenesis: a doubleedged sword. Mol Cancer. 2018;17:101.

17. Sun $T, W u R$, Ming L. The role of m6A RNA methylation in cancer. Biomedicine pharmacotherapy $=$ Biomedecine pharmacotherapie. 2019;112:108613.

18. Cheng $M$, et al. The $\mathrm{m}(6) A$ methyltransferase METTL3 promotes bladder cancer progression via AFF4/NF-kappaB/MYC signaling network. Oncogene. 2019;38:3667-80. 
19. Jin $\mathrm{H}$, et al. $\mathrm{N}(6)$-methyladenosine modification of ITGA6 mRNA promotes the development and progression of bladder cancer. EBioMedicine. 2019;47:195-207.

20. Kreso A, Dick JE. Evolution of the cancer stem cell model. Cell stem cell. 2014;14:275-91.

21. Kurtova AV, et al. Blocking PGE2-induced tumour repopulation abrogates bladder cancer chemoresistance. Nature. 2015;517:209-13.

22. Mazzieri R, et al. Targeting the ANG2/TIE2 axis inhibits tumor growth and metastasis by impairing angiogenesis and disabling rebounds of proangiogenic myeloid cells. Cancer cell. 2011;19:512-26.

23. Goel HL, Mercurio AM. VEGF targets the tumour cell. Nat Rev Cancer. 2013;13:871-82.

24. Randall LM, et al. Markers of angiogenesis in high-risk, early-stage cervical cancer: A Gynecologic Oncology Group study. Gynecol Oncol. 2009;112:583-9.

25. Tanaka F, et al. Evaluation of angiogenesis in non-small cell lung cancer: comparison between antiCD34 antibody and anti-CD105 antibody. Clinical cancer research: an official journal of the American Association for Cancer Research. 2001;7:3410-5.

26. Liu L, et al. METTL3 Promotes Tumorigenesis and Metastasis through BMI1 m(6)A Methylation in Oral Squamous Cell Carcinoma. Molecular therapy: the journal of the American Society of Gene Therapy. 2020;28:2177-90.

27. Dominissini D, Moshitch-Moshkovitz S, Salmon-Divon M, Amariglio N, Rechavi G. Transcriptomewide mapping of $\mathrm{N}(6)$-methyladenosine by $\mathrm{m}(6) \mathrm{A}$-seq based on immunocapturing and massively parallel sequencing. Nature protocols. 2013;8:176-89.

28. Johnson DT, et al. Conditional Expression of the Androgen Receptor Increases Susceptibility of Bladder Cancer in Mice. PloS one. 2016;11:e0148851.

29. Irving CC, Murphy WM, Daniel DS. Comparative carcinogenicity of N-butyl-N-(3-carboxypropyl)nitrosamine and N-butyl-N-(4-hydroxybutyl)nitrosamine for the urinary bladder of (C57BL/6 X DBA/2)F1 mice. J Natl Cancer Inst. 1984;73:753-6.

30. Li T, et al. METTL3 facilitates tumor progression via an m(6)A-IGF2BP2-dependent mechanism in colorectal carcinoma. Mol Cancer. 2019;18:112.

31. Choe J, et al. mRNA circularization by METTL3-elF3h enhances translation and promotes oncogenesis. Nature. 2018;561:556-60.

32. Liu J, et al. m(6)A mRNA methylation regulates AKT activity to promote the proliferation and tumorigenicity of endometrial cancer. Nat Cell Biol. 2018;20:1074-83.

33. Barbieri I, et al. Promoter-bound METTL3 maintains myeloid leukaemia by m(6)A-dependent translation control. Nature. 2017;552:126-31.

34. Yue B, et al. METTL3-mediated N6-methyladenosine modification is critical for epithelialmesenchymal transition and metastasis of gastric cancer. Mol Cancer. 2019;18:142.

35. Gengenbacher N, et al Timed Ang2-targeted therapy identifies the Angiopoietin-Tie pathway as key regulator of fatal lymphogenous metastasis. Cancer discovery (2020). 
36. Saito R, et al. Molecular Subtype-Specific Immunocompetent Models of High-Grade Urothelial Carcinoma Reveal Differential Neoantigen Expression and Response to Immunotherapy. Cancer research. 2018;78:3954-68.

37. Papafotiou G, et al. KRT14 marks a subpopulation of bladder basal cells with pivotal role in regeneration and tumorigenesis. Nature communications. 2016;7:11914.

\section{Figures}
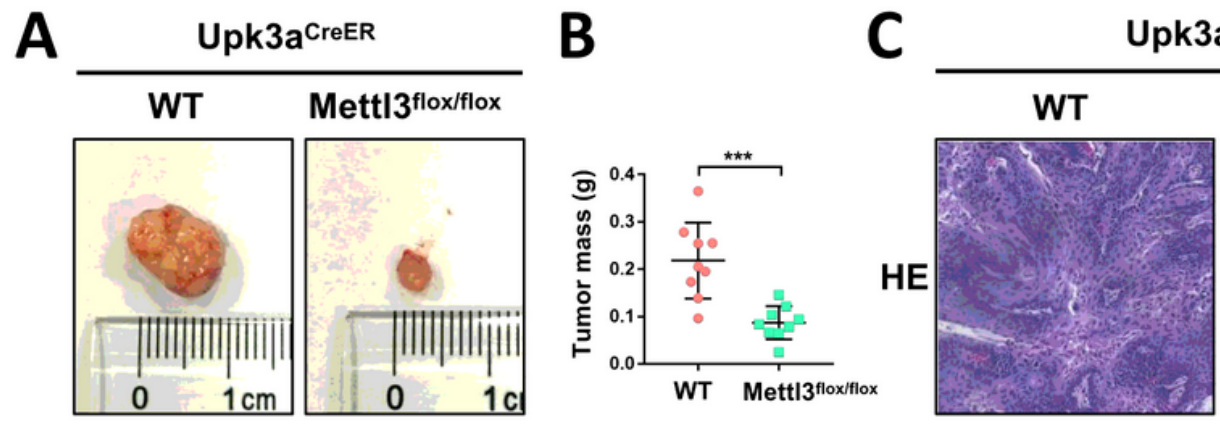

D

Mettl3 flox/flox
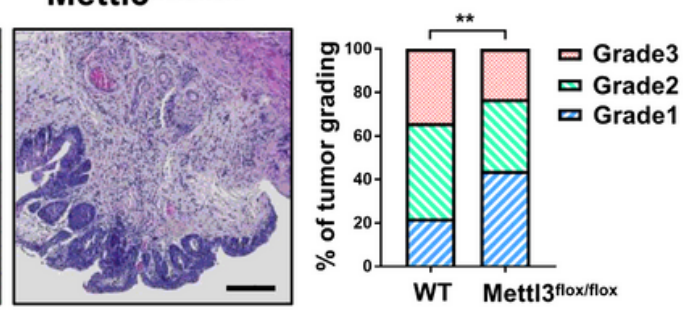

$\mathbf{E}$

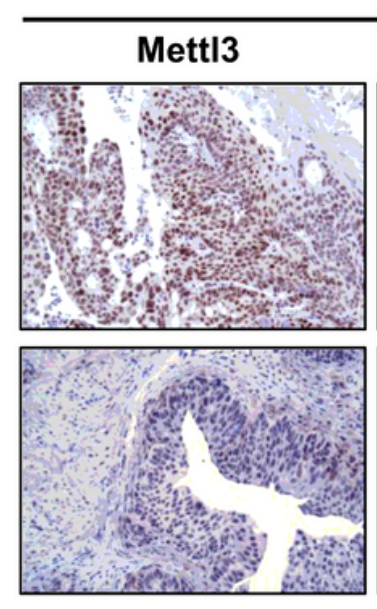

Upk3aCreER

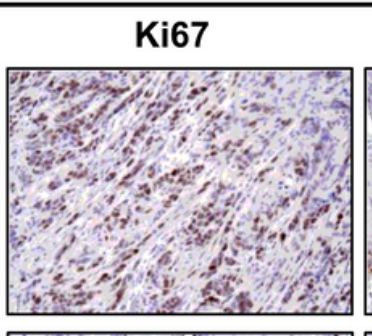

\section{Caspase-3}

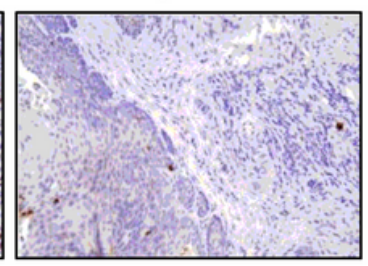

WT
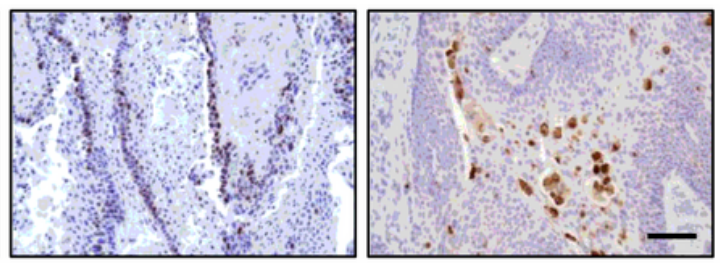

Mettl/3flox/flox

$\mathbf{F}$
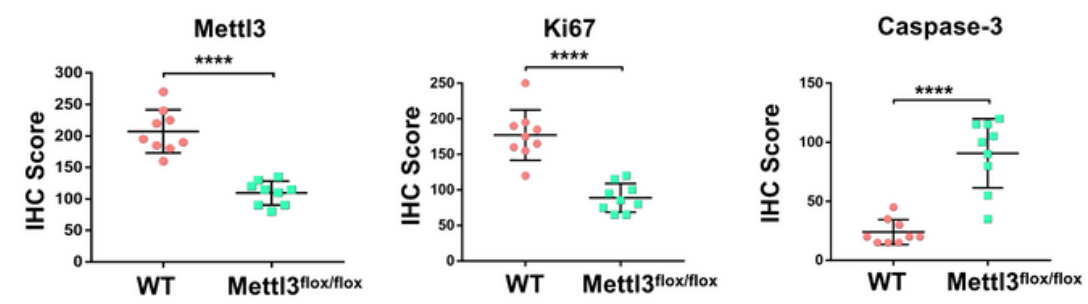

\section{Figure 1}

Knockout of Mett|3 in urothelium inhibits the development of BCa A. Representative images of BCa lesions from Upk3aCreER, Mettl3wt/wt or Upk3aCreER, Mettl3flox/flox mice those 26 weeks after BBN 
treatment of at least three independent groups. B. Statistics of bladder weight of mice in two groups. C. Representative image of H\&E staining of BCa section in wild type and Mettl3flox/flox mice. Tamoxifen was intraperitoneal injected into BBN-induced Upk3aCreER wild type and Mettl3flox/flox mice. Bladders were harvested after 24 hours tamoxifen treatment. Scale bar, $100 \mu \mathrm{m}$. D. Statistics of tumor grading result was based on the H\&E staining experiment. E. Immumohistochemical staining for the expression pattern of Upk3a, Mettl3, Ki67 and Caspase-3 in both wild type and Mettl3flox/flox mice. Scale bar, 100 $\mu \mathrm{m}$. F. Immunohistochemical staining scores of Mettl3, Ki67 and Caspase-3 were counted respectively.
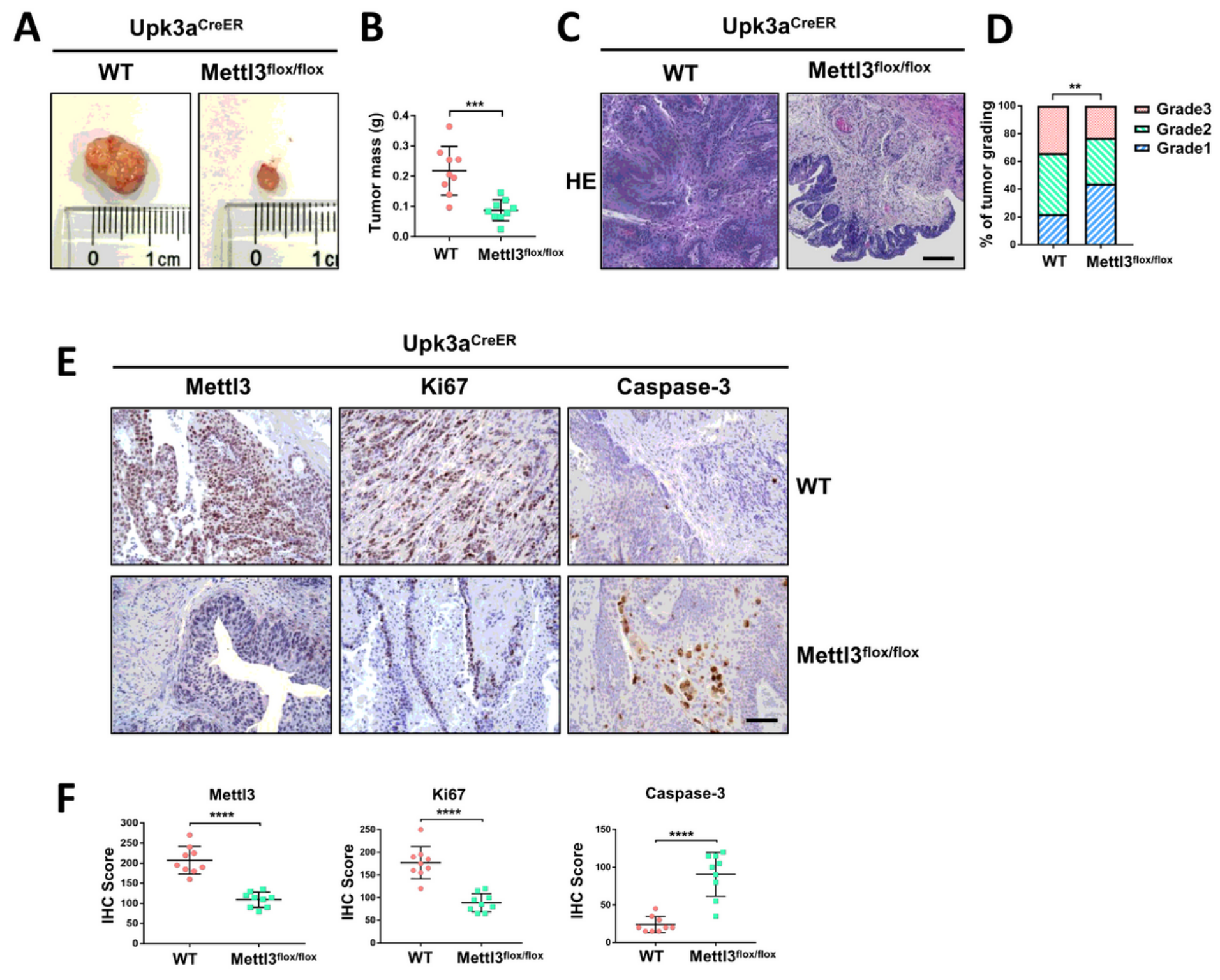

\section{Figure 1}

Knockout of Mettl3 in urothelium inhibits the development of BCa A. Representative images of BCa lesions from Upk3aCreER, Mettl3wt/wt or Upk3aCreER, Mettl3flox/flox mice those 26 weeks after BBN treatment of at least three independent groups. B. Statistics of bladder weight of mice in two groups. C. 
Representative image of H\&E staining of BCa section in wild type and Mettl3flox/flox mice. Tamoxifen was intraperitoneal injected into BBN-induced Upk3aCreER wild type and Mettl3flox/flox mice. Bladders were harvested after 24 hours tamoxifen treatment. Scale bar, $100 \mu \mathrm{m}$. D. Statistics of tumor grading result was based on the H\&E staining experiment. E. Immumohistochemical staining for the expression pattern of Upk3a, Mettl3, Ki67 and Caspase-3 in both wild type and Mettl3flox/flox mice. Scale bar, 100 $\mu \mathrm{m}$. F. Immunohistochemical staining scores of Mett|3, Ki67 and Caspase-3 were counted respectively.
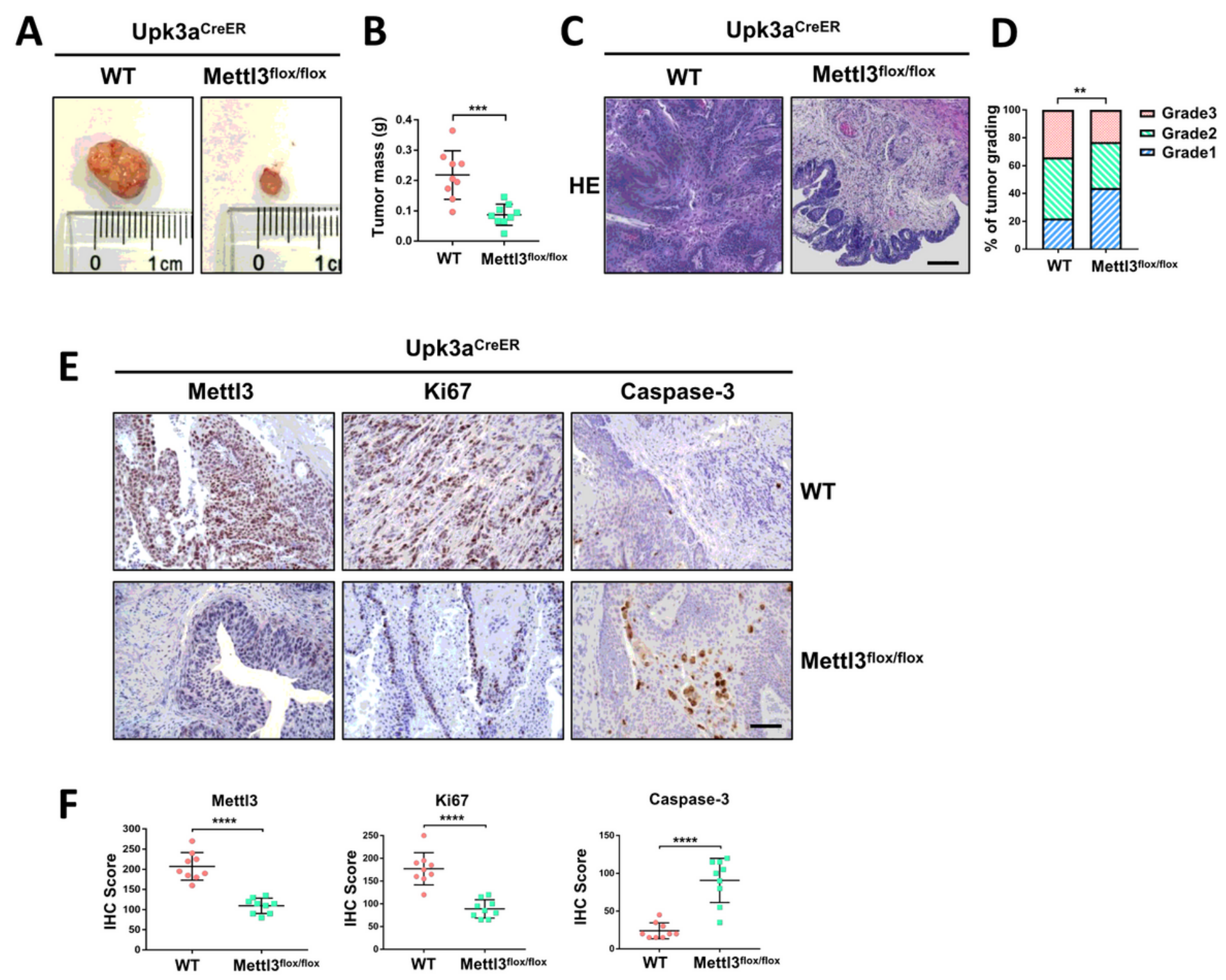

\section{Figure 1}

Knockout of Mettl3 in urothelium inhibits the development of BCa A. Representative images of BCa lesions from Upk3aCreER, Mett|3wt/wt or Upk3aCreER, Mettl3flox/flox mice those 26 weeks after BBN treatment of at least three independent groups. B. Statistics of bladder weight of mice in two groups. C. Representative image of H\&E staining of BCa section in wild type and Mettl3flox/flox mice. Tamoxifen 
was intraperitoneal injected into BBN-induced Upk3aCreER wild type and Mettl3flox/flox mice. Bladders were harvested after 24 hours tamoxifen treatment. Scale bar, $100 \mu \mathrm{m}$. D. Statistics of tumor grading result was based on the H\&E staining experiment. E. Immumohistochemical staining for the expression pattern of Upk3a, Mettl3, Ki67 and Caspase-3 in both wild type and Mettl3flox/flox mice. Scale bar, 100 $\mu \mathrm{m}$. F. Immunohistochemical staining scores of Mettl3, Ki67 and Caspase-3 were counted respectively.
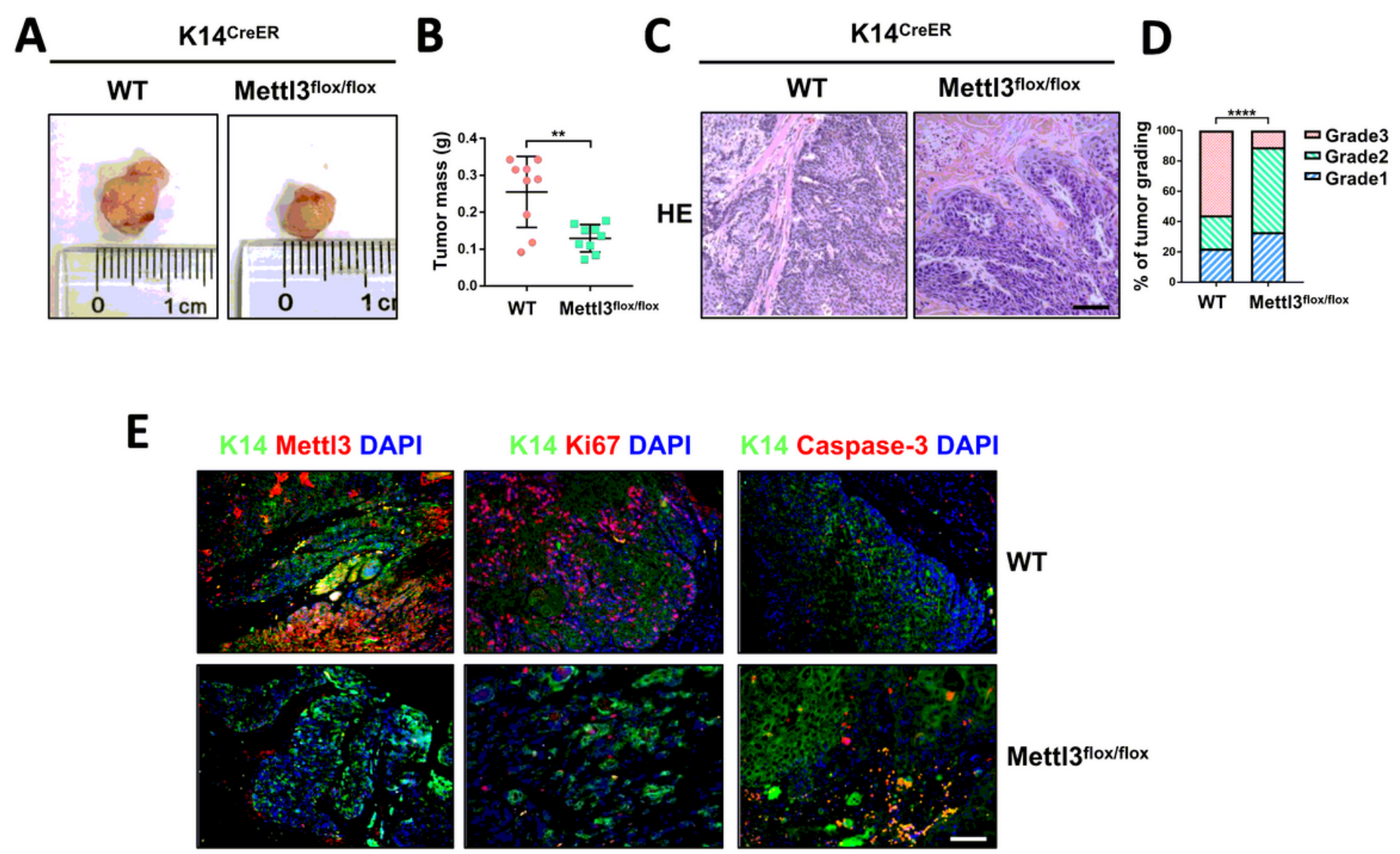

Mett|3flox/flox

$\mathbf{F}$
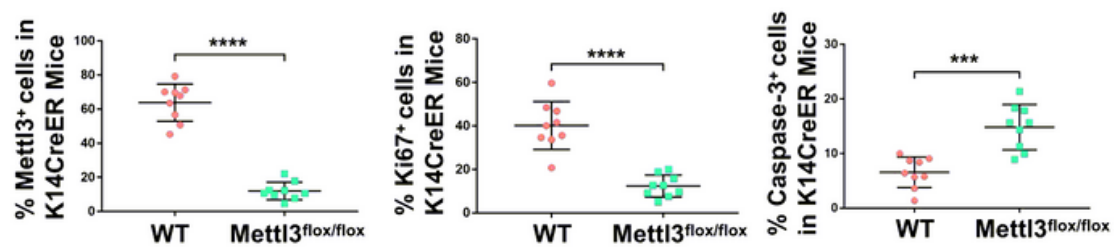

\section{Figure 2}

Deletion of Mettl3 in K14+ cells displays reduced tumorigenicity, cell proliferation and survival. A. Representative images of BCa lesions from K14CreER, Mettl3wt/wt or K14CreER, Mettl3flox/flox mice. B. The bladder weight was calculated in wild type and Mettl3flox/flox mice respectively. C. Representative H\&E staining images of BCa section in wild type and Mettl3flox/flox mice show the histological atypia. Scale bars $50 \mu \mathrm{m}$. Scale bar, $100 \mu \mathrm{m}$. D. Assessment of malignancy in wild type and Mettl3flox/flox mice 
by tumor grading. E. Immunofluorescent staining was used to evaluate the expression of K14, Mettl3 (left), Ki67 (middle) and Caspase-3 (right). DAPI staining was used to mark nucleus. Scale bar, $100 \mu \mathrm{m}$. F. Calculation of Mettl3 (left), Ki67 (middle) and Caspase-3 (right) positive cells in wild type and K14CreER, Mett|3flox/flox mice
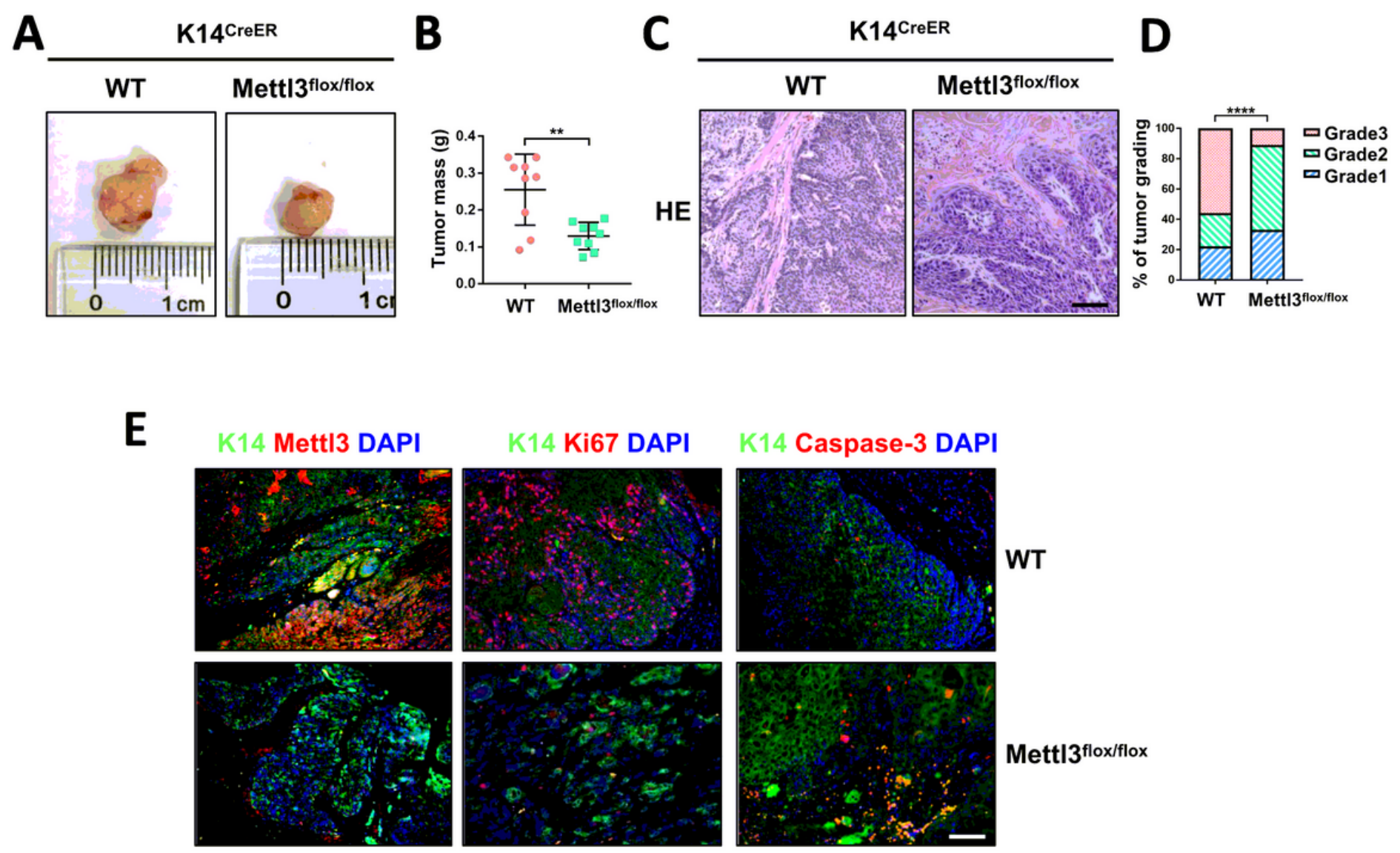

\section{Mettl/ $3^{\text {flox/flox }}$}

$\mathbf{F}$
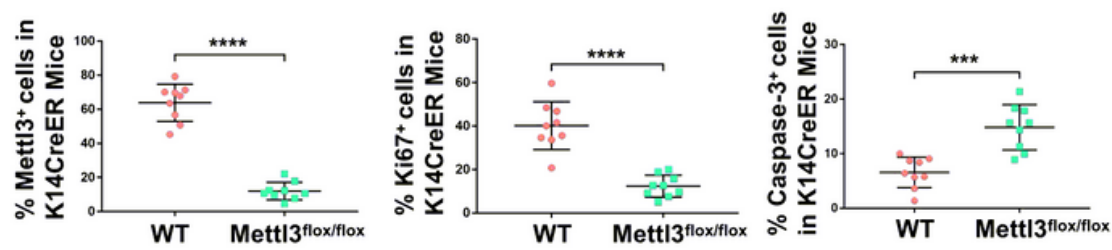

\section{Figure 2}

Deletion of Mettl3 in K14+ cells displays reduced tumorigenicity, cell proliferation and survival. A. Representative images of BCa lesions from K14CreER, Mettl3wt/wt or K14CreER, Mettl3flox/flox mice. B. The bladder weight was calculated in wild type and Mettl3flox/flox mice respectively. C. Representative H\&E staining images of BCa section in wild type and Mettl3flox/flox mice show the histological atypia. Scale bars $50 \mu \mathrm{m}$. Scale bar, $100 \mu \mathrm{m}$. D. Assessment of malignancy in wild type and Mettl3flox/flox mice by tumor grading. E. Immunofluorescent staining was used to evaluate the expression of K14, Mettl3 
(left), Ki67 (middle) and Caspase-3 (right). DAPI staining was used to mark nucleus. Scale bar, $100 \mu \mathrm{m}$. F. Calculation of Mettl3 (left), Ki67 (middle) and Caspase-3 (right) positive cells in wild type and K14CreER, Mettl3flox/flox mice
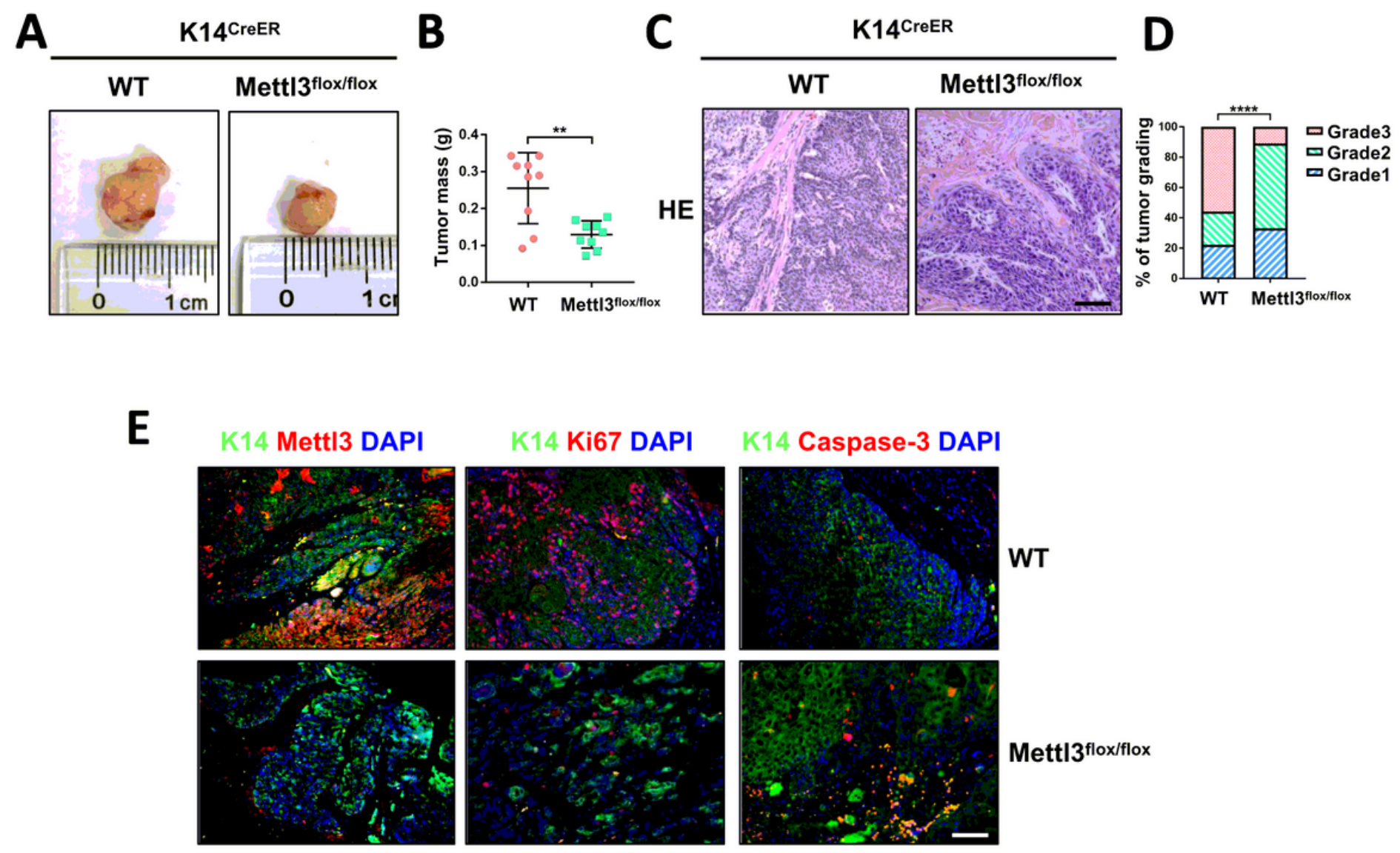

\section{Mettl3flox/flox}

F
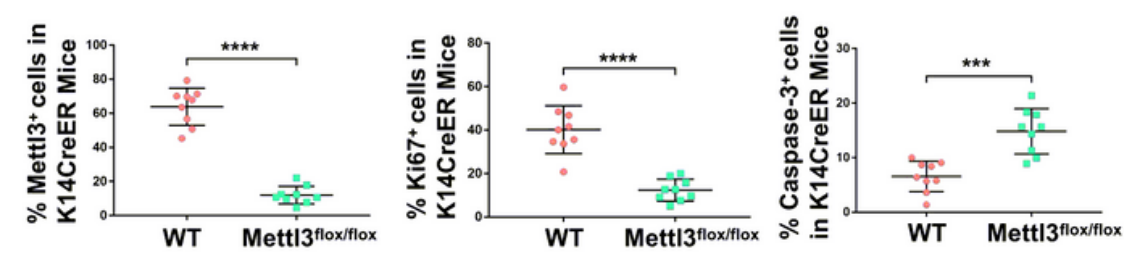

\section{Figure 2}

Deletion of Mettl3 in K14+ cells displays reduced tumorigenicity, cell proliferation and survival. A. Representative images of BCa lesions from K14CreER, Mettl3wt/wt or K14CreER, Mettl3flox/flox mice. B. The bladder weight was calculated in wild type and Mettl3flox/flox mice respectively. C. Representative H\&E staining images of BCa section in wild type and Mettl3flox/flox mice show the histological atypia. Scale bars $50 \mu \mathrm{m}$. Scale bar, $100 \mu \mathrm{m}$. D. Assessment of malignancy in wild type and Mettl3flox/flox mice by tumor grading. E. Immunofluorescent staining was used to evaluate the expression of K14, Mettl3 (left), Ki67 (middle) and Caspase-3 (right). DAPI staining was used to mark nucleus. Scale bar, $100 \mu \mathrm{m}$. F. 
Calculation of Mettl3 (left), Ki67 (middle) and Caspase-3 (right) positive cells in wild type and K14CreER, Mettl3flox/flox mice
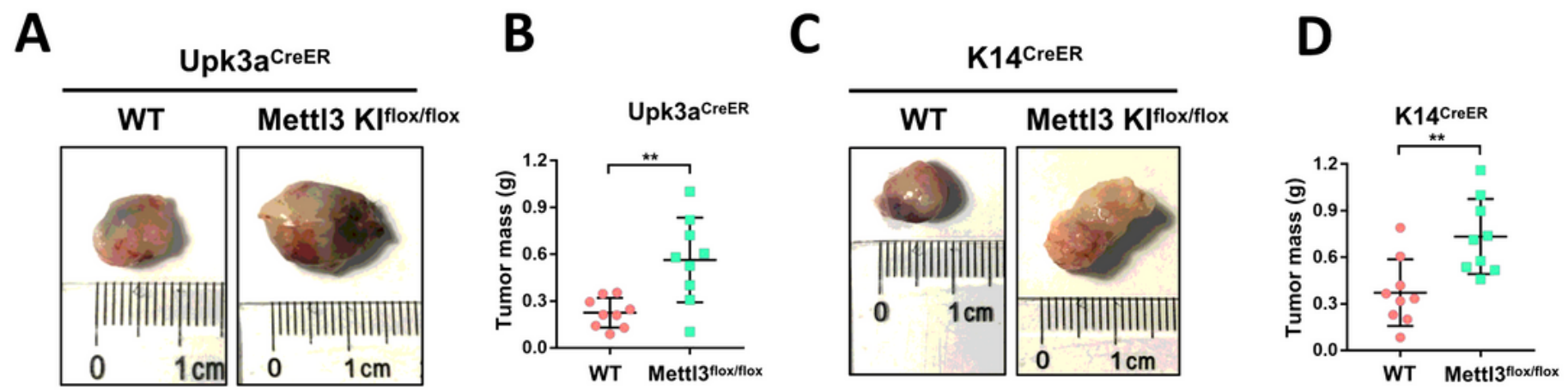

E
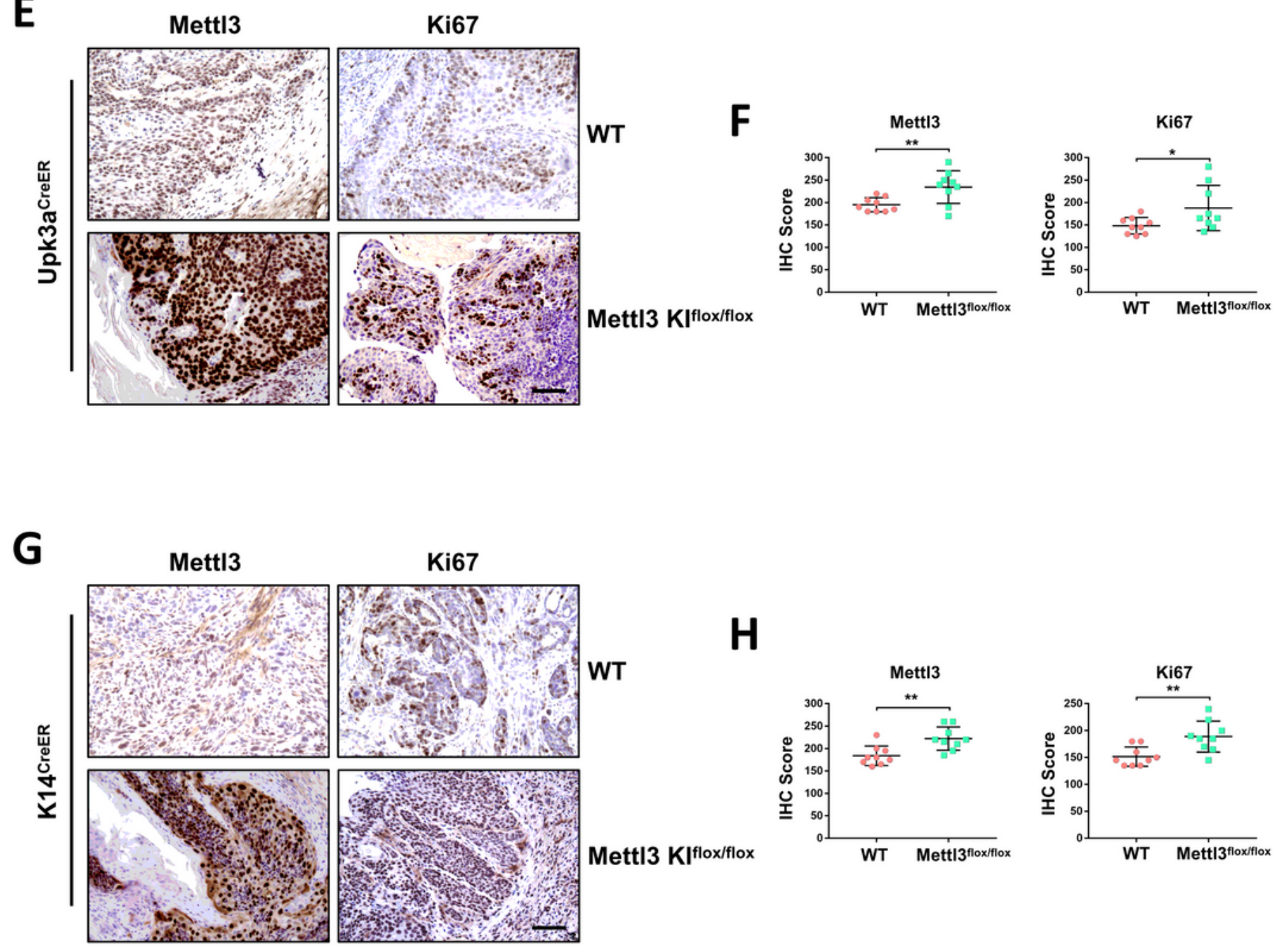

Figure 3

Overexpression of Mettl3 in urothelium or $\mathrm{K} 14+$ cells promotes $\mathrm{BCa}$ progression in vivo. A. Representative images of BCa in Upk3aCreER WT (left) and Upk3aCreER Mettl3 KI (right) mice showing the tumor volume. B. Measurement of tumor mass in Upk3a-specific WT and Mettl3 KI groups respectively. C. 
Tumor volume was shown in K14-specific WT (left) and KI (right) mice. D. The weight of tumor collected from K14CreER wild type and K14CreER Mettl3 KI mice was calculated respectively. E. Immunostaining was applied to explore the expression level of Mettl3 and Ki67 in Upk3aCreER WT or Mettl3 KI mice. Scale bars $100 \mu \mathrm{m}$. F. IHC scoring was calculated based on the statistics of staining intensity and ratio of positive cells above. G. Representative IHC staining images of Mettl3 or Ki67 in K14CreER wild type and K14CreER Mettl3 KI mice. Scale bars $100 \mu \mathrm{m}$. H. Calculation of IHC scores according to the immunostaining images above.

A

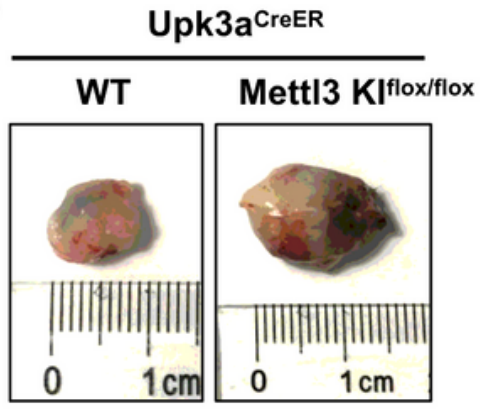

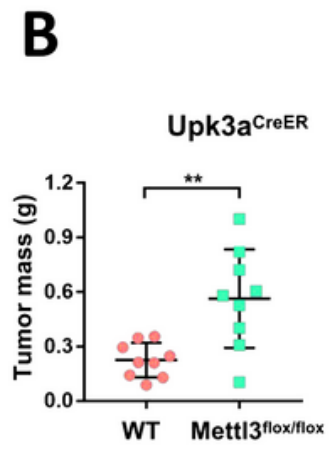

C

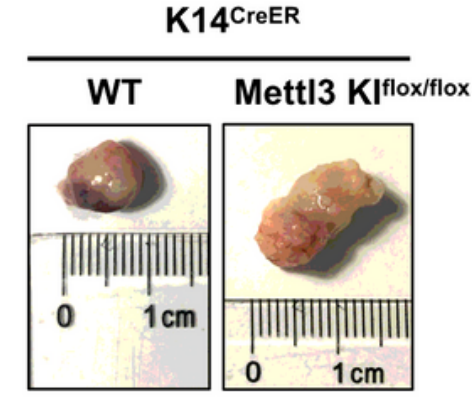

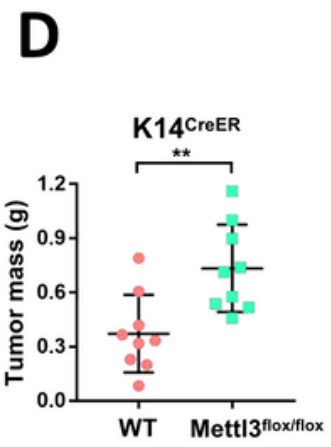

E

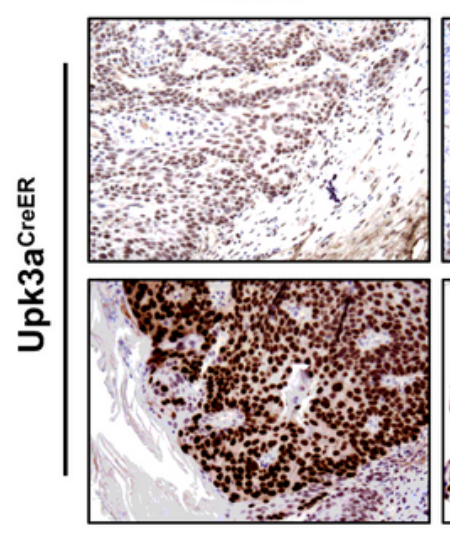

G

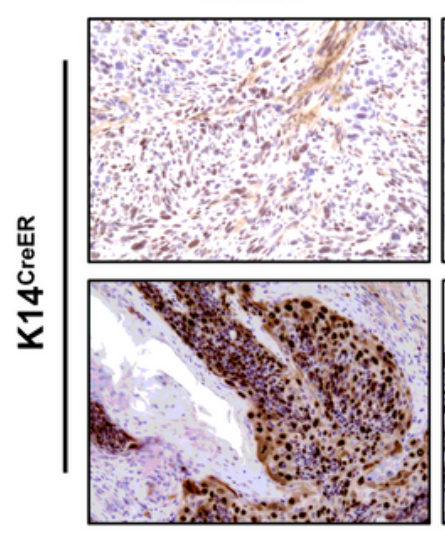

Ki67

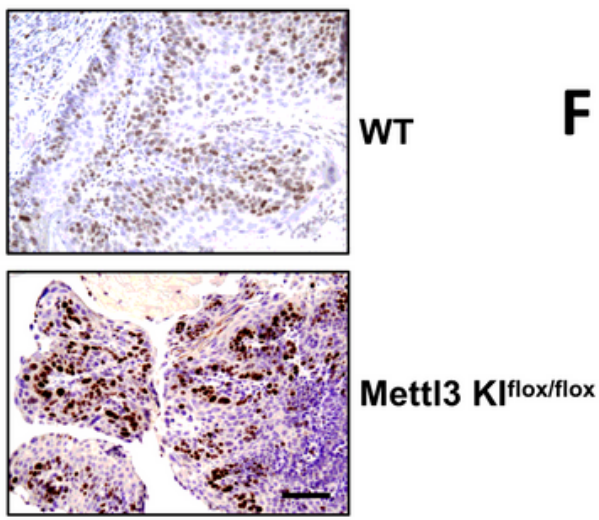

F
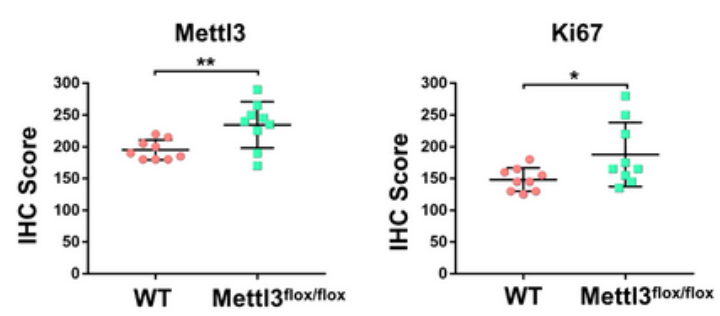

H

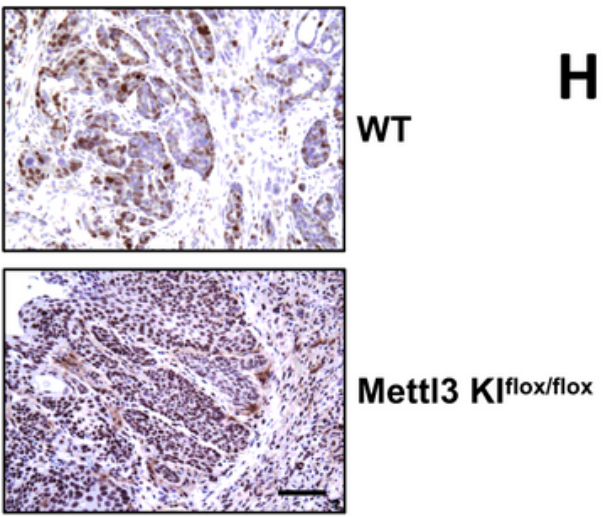

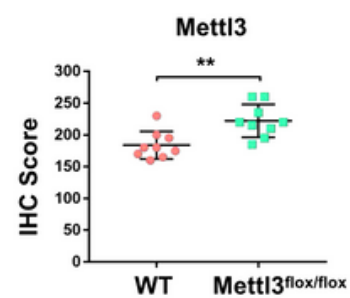

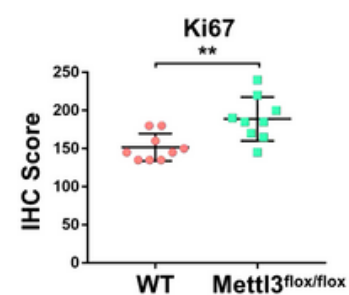




\section{Figure 3}

Overexpression of Mettl3 in urothelium or $\mathrm{K} 14+$ cells promotes BCa progression in vivo. A. Representative images of BCa in Upk3aCreER WT (left) and Upk3aCreER Mettl3 KI (right) mice showing the tumor volume. B. Measurement of tumor mass in Upk3a-specific WT and Mettl3 KI groups respectively. C. Tumor volume was shown in K14-specific WT (left) and KI (right) mice. D. The weight of tumor collected from K14CreER wild type and K14CreER Mettl3 KI mice was calculated respectively. E. Immunostaining was applied to explore the expression level of Mettl3 and Ki67 in Upk3aCreER WT or Mettl3 KI mice. Scale bars $100 \mu \mathrm{m}$. F. IHC scoring was calculated based on the statistics of staining intensity and ratio of positive cells above. G. Representative IHC staining images of Mettl3 or Ki67 in K14CreER wild type and K14CreER Mettl3 KI mice. Scale bars $100 \mu \mathrm{m}$. H. Calculation of IHC scores according to the immunostaining images above. 
A

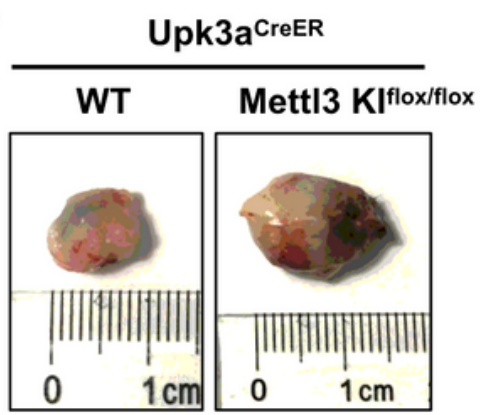

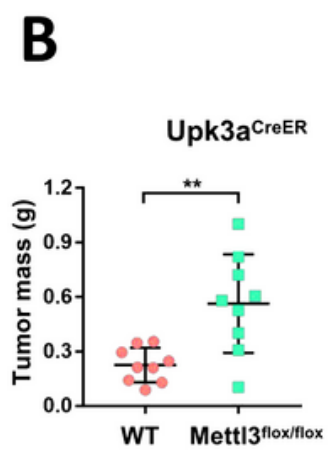

C

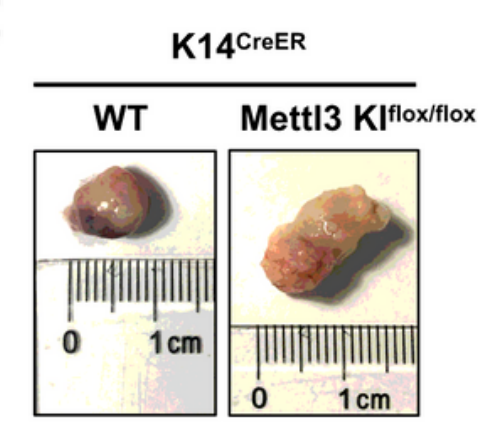

D

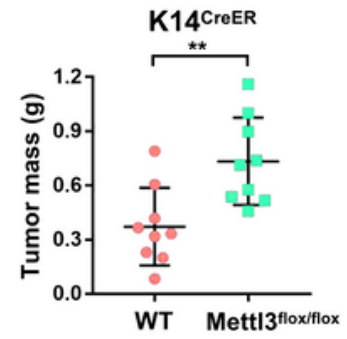

E
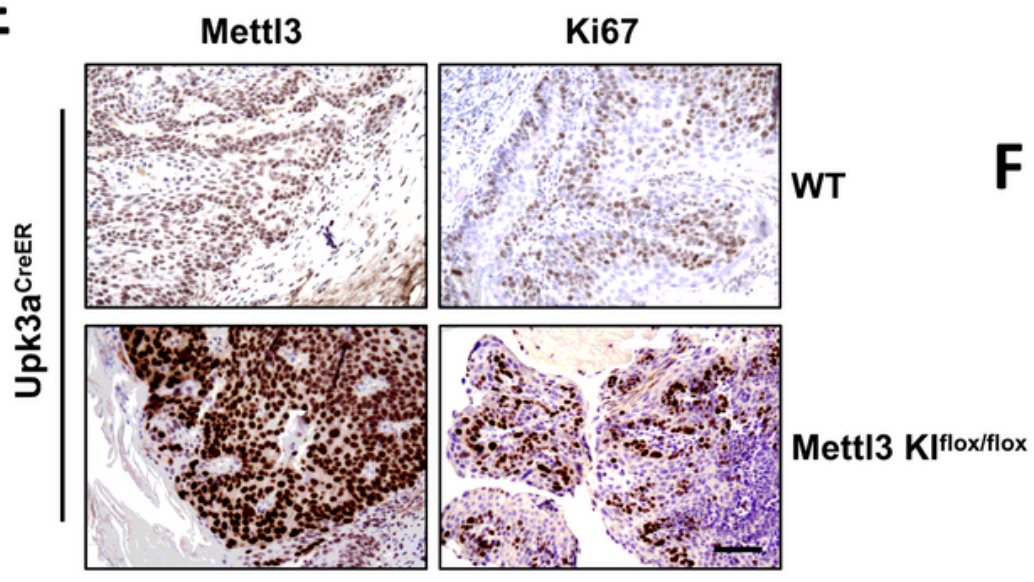

F
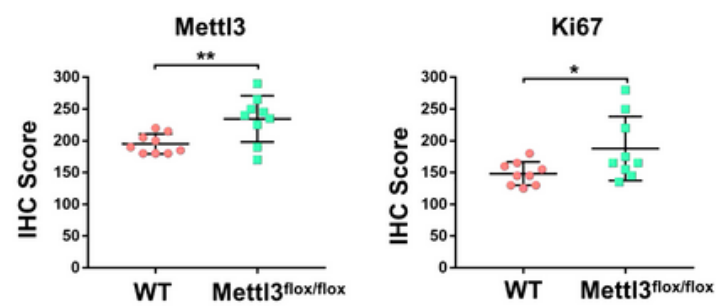

G

Mett|3

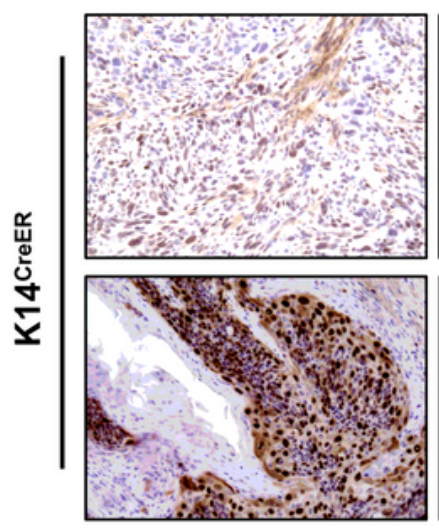

Ki67

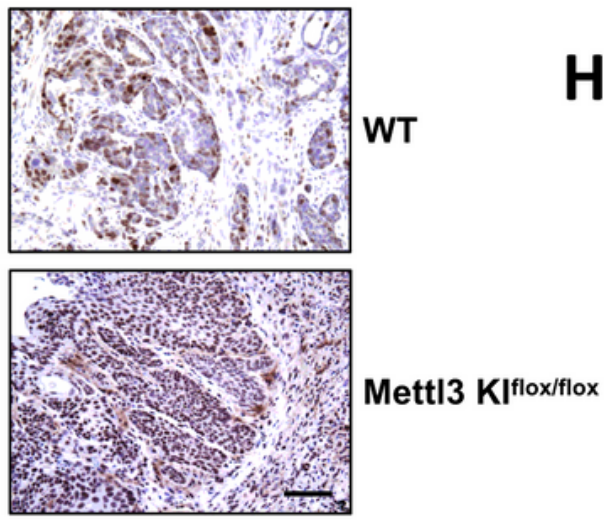

H

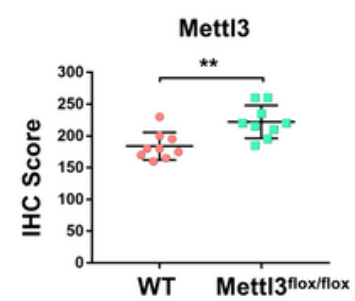

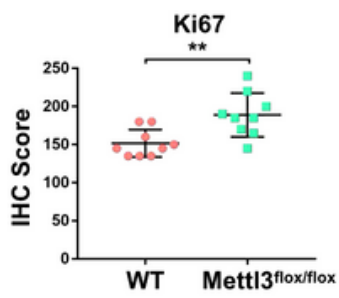

\section{Figure 3}

Overexpression of Mettl3 in urothelium or K14+ cells promotes BCa progression in vivo. A. Representative images of BCa in Upk3aCreER WT (left) and Upk3aCreER Mettl3 KI (right) mice showing the tumor volume. B. Measurement of tumor mass in Upk3a-specific WT and Mettl3 KI groups respectively. C. Tumor volume was shown in K14-specific WT (left) and KI (right) mice. D. The weight of tumor collected from K14CreER wild type and K14CreER Mettl3 KI mice was calculated respectively. E. Immunostaining 
was applied to explore the expression level of Mettl3 and Ki67 in Upk3aCreER WT or Mettl3 KI mice. Scale bars $100 \mu \mathrm{m}$. F. IHC scoring was calculated based on the statistics of staining intensity and ratio of positive cells above. G. Representative IHC staining images of Mettl3 or Ki67 in K14CreER wild type and K14CreER Mettl3 KI mice. Scale bars $100 \mu \mathrm{m}$. H. Calculation of IHC scores according to the immunostaining images above.

A

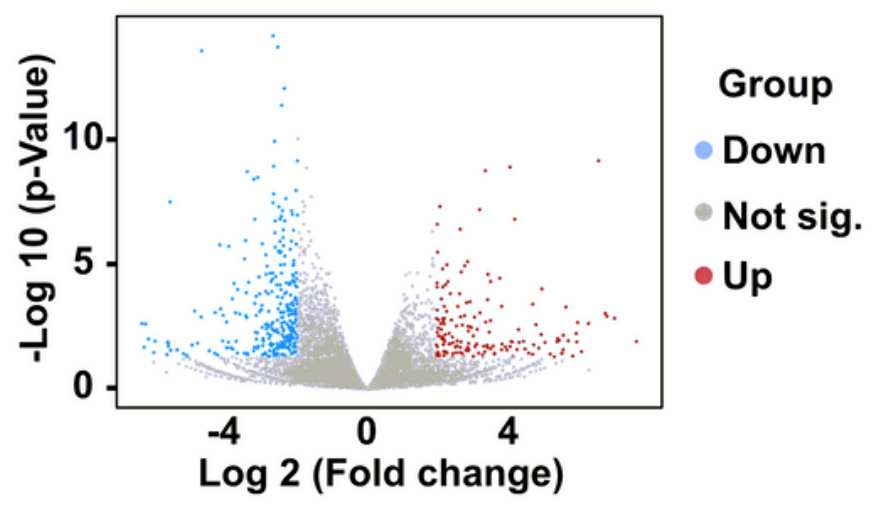

B

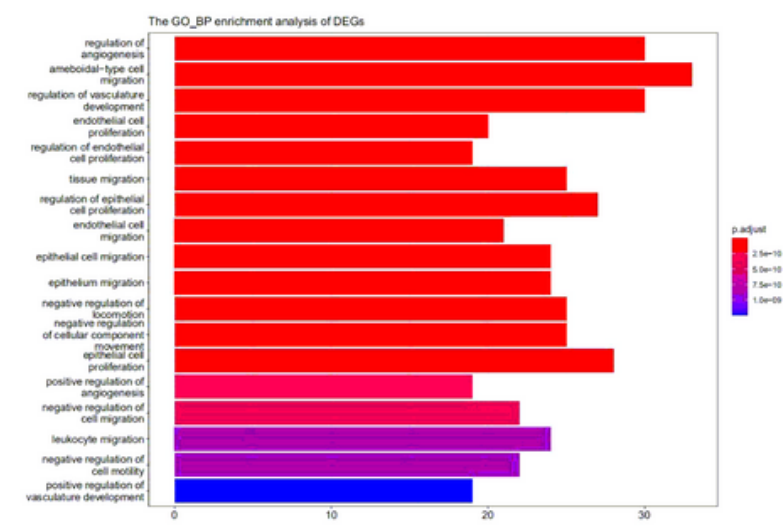

C

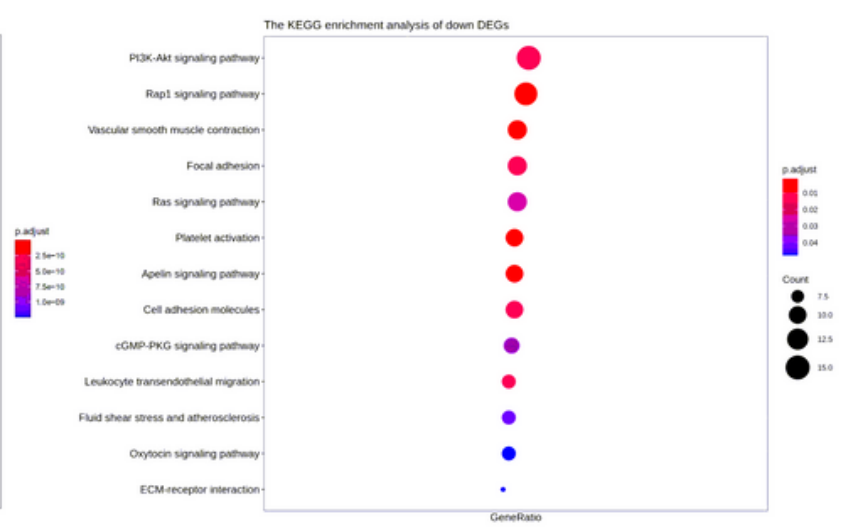

D

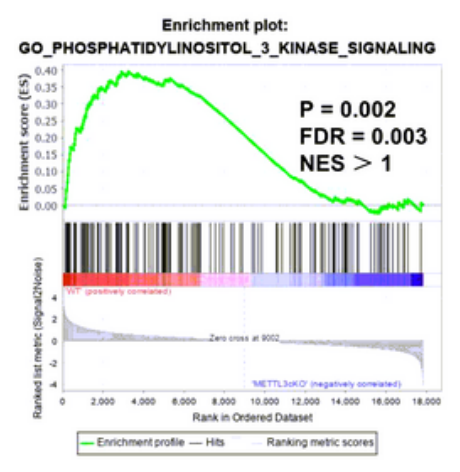

$\mathbf{E}$

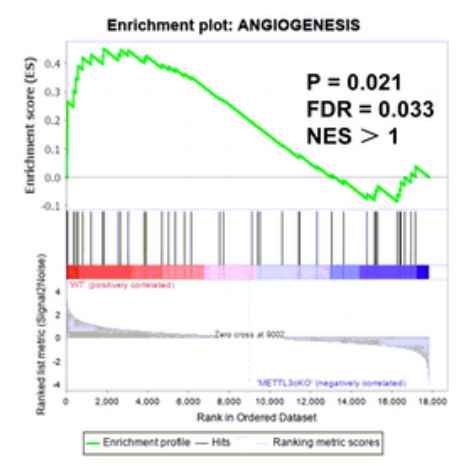

$\mathbf{F}$

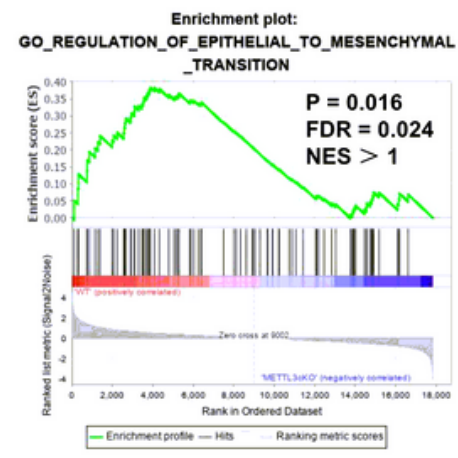

Figure 4 
RNA-sequencing reveals Mettl3 regulates PI3K pathway and angiogenesis in $\mathrm{BCa} A$. The DEGs were shown by volcano scatterplot. Blue dots represented downregulated genes by deletion of Mettl3, gray for not significantly changed and the red one for the upregulated genes. B. The Gene Ontology Biological Process enrichment analysis of DEGs identified the most relevant function modulated by Mett|3. C. The KEGG enrichment analysis was conducted to examine downstream signaling pathway by monitoring down DEGs. D. Gene Set Enrichment Assay plots of GO phosphatidylinositol 3 kinase signaling for Mett|3-regulated genes from RNA-seq datasets. E. Gene Set Enrichment Assay plots of angiogenesis for Mett|3-regulated genes from RNA-seq data. F. Gene Set Enrichment Assay plots of GO regulation of epithelial to mesenchymal transition for Mett|3-regulated genes from RNA-seq datasets. 
A

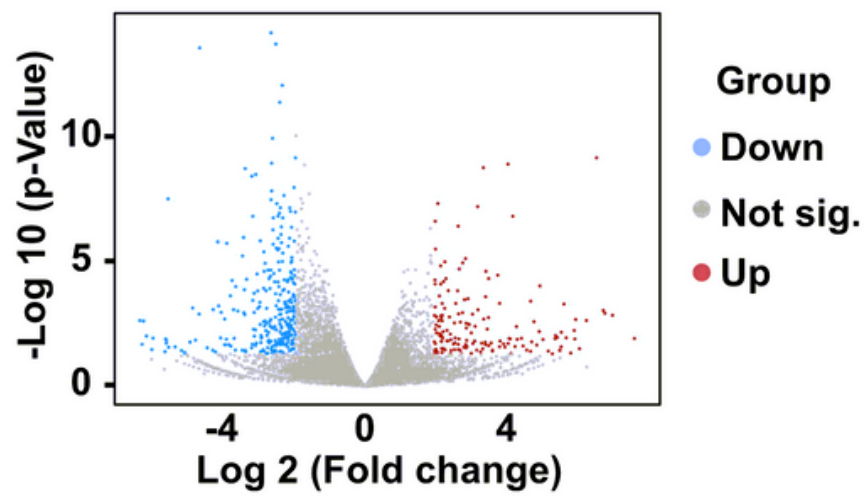

B

C
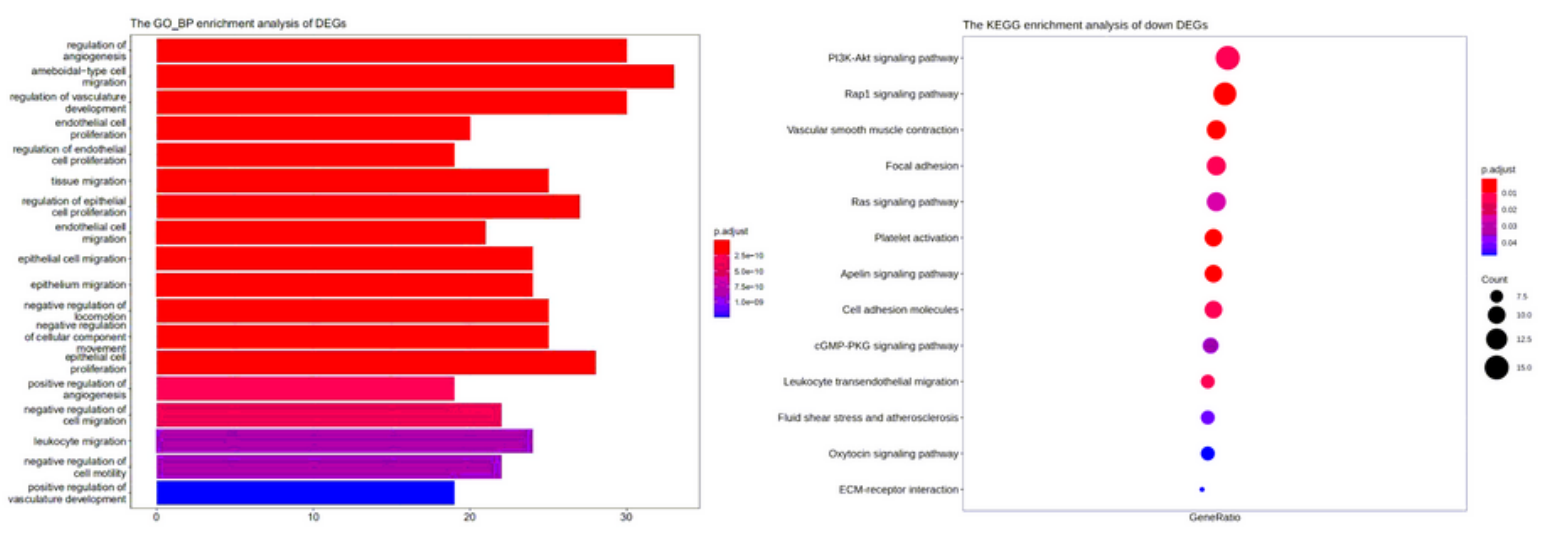

D

E
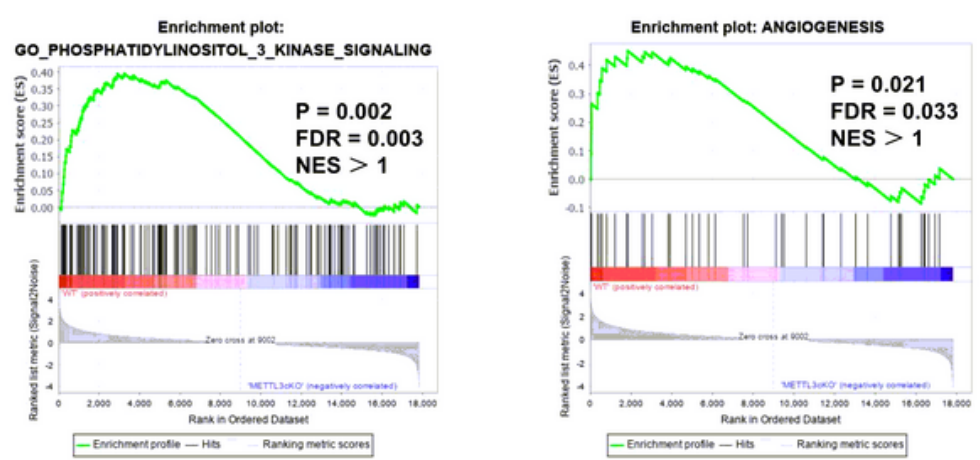

$\mathbf{F}$

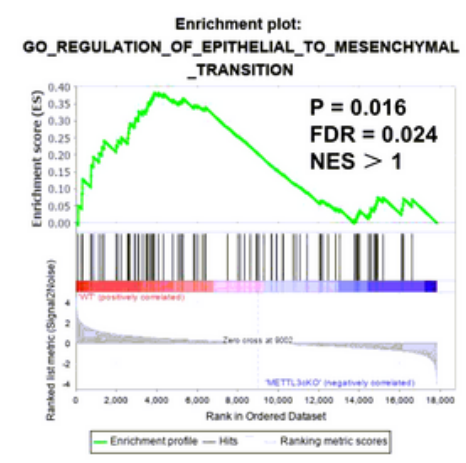

\section{Figure 4}

RNA-sequencing reveals Mettl3 regulates PI3K pathway and angiogenesis in $\mathrm{BCa} A$. The DEGs were shown by volcano scatterplot. Blue dots represented downregulated genes by deletion of Mettl3, gray for not significantly changed and the red one for the upregulated genes. B. The Gene Ontology Biological Process enrichment analysis of DEGs identified the most relevant function modulated by Mettl3. $\mathrm{C}$. The KEGG enrichment analysis was conducted to examine downstream signaling pathway by monitoring 
down DEGs. D. Gene Set Enrichment Assay plots of GO phosphatidylinositol 3 kinase signaling for Mett|3-regulated genes from RNA-seq datasets. E. Gene Set Enrichment Assay plots of angiogenesis for Mett|3-regulated genes from RNA-seq data. F. Gene Set Enrichment Assay plots of GO regulation of epithelial to mesenchymal transition for Mettl3-regulated genes from RNA-seq datasets.

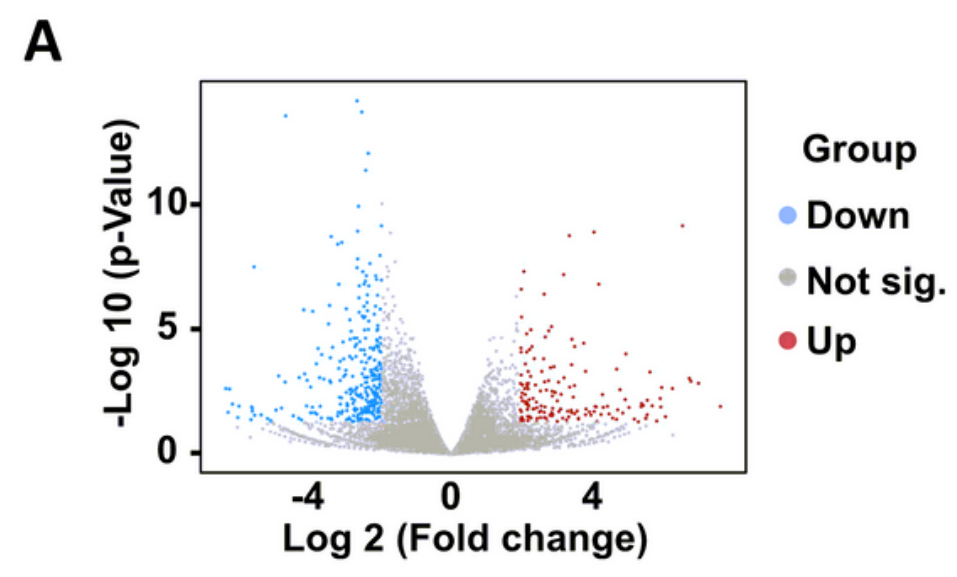

B

C

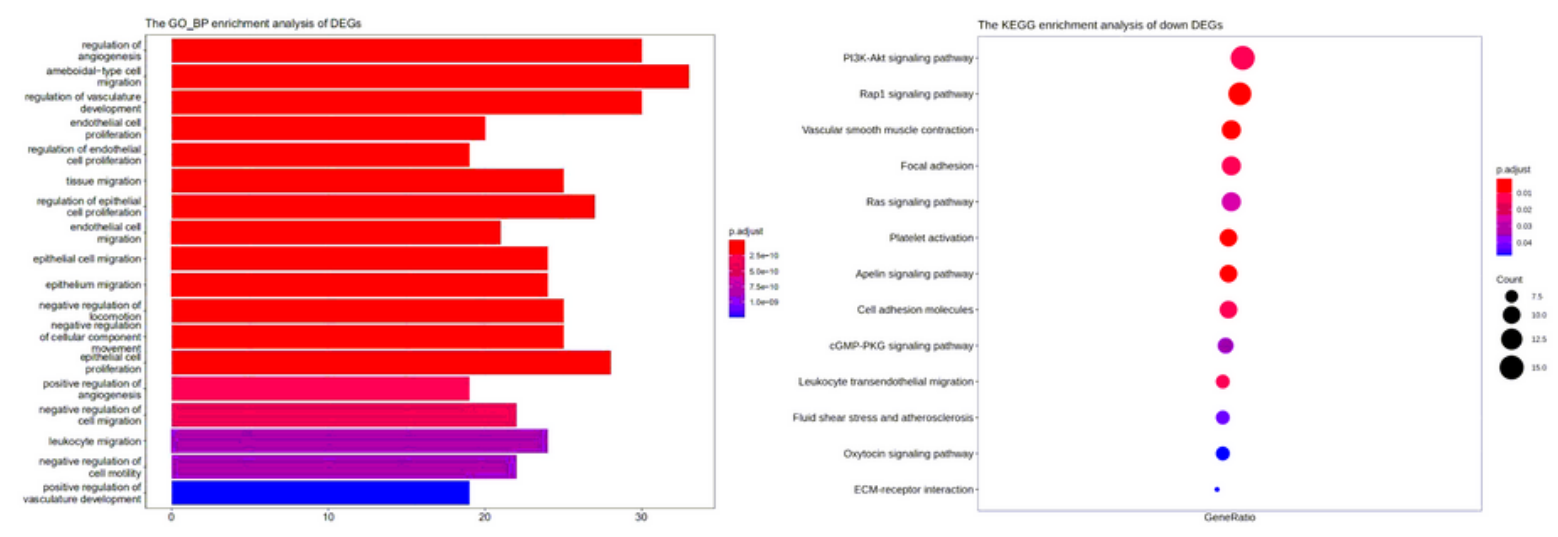

D

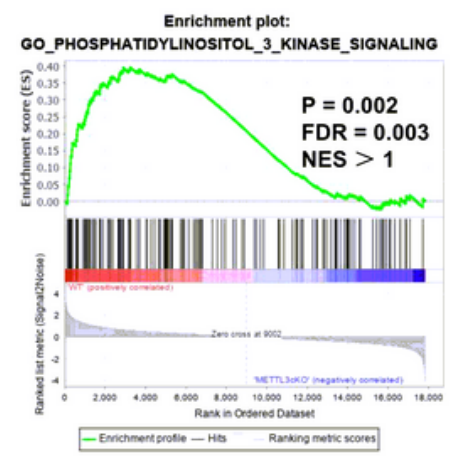

$\mathbf{E}$

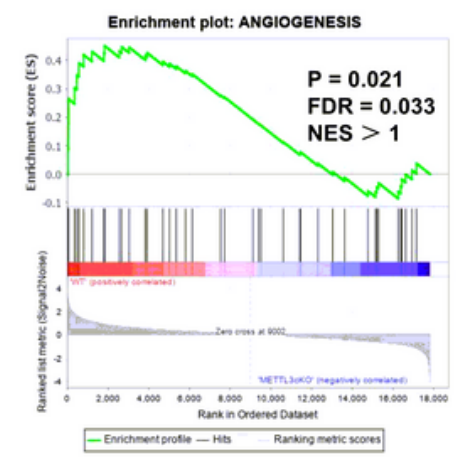

$\mathbf{F}$

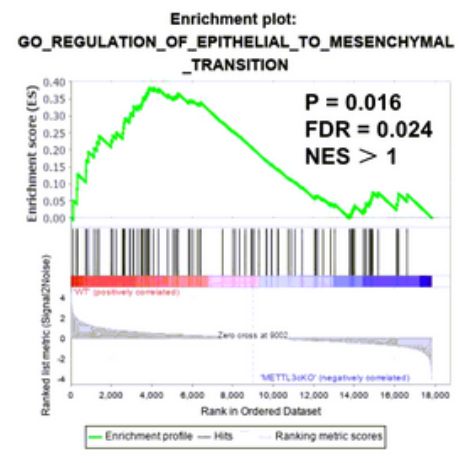

Figure 4 
RNA-sequencing reveals Mettl3 regulates PI3K pathway and angiogenesis in $\mathrm{BCa} A$. The DEGs were shown by volcano scatterplot. Blue dots represented downregulated genes by deletion of Mettl3, gray for not significantly changed and the red one for the upregulated genes. B. The Gene Ontology Biological Process enrichment analysis of DEGs identified the most relevant function modulated by Mettl3. $\mathrm{C}$. The KEGG enrichment analysis was conducted to examine downstream signaling pathway by monitoring down DEGs. D. Gene Set Enrichment Assay plots of GO phosphatidylinositol 3 kinase signaling for Mett|3-regulated genes from RNA-seq datasets. E. Gene Set Enrichment Assay plots of angiogenesis for Mett|3-regulated genes from RNA-seq data. F. Gene Set Enrichment Assay plots of GO regulation of epithelial to mesenchymal transition for Mett|3-regulated genes from RNA-seq datasets. 
A

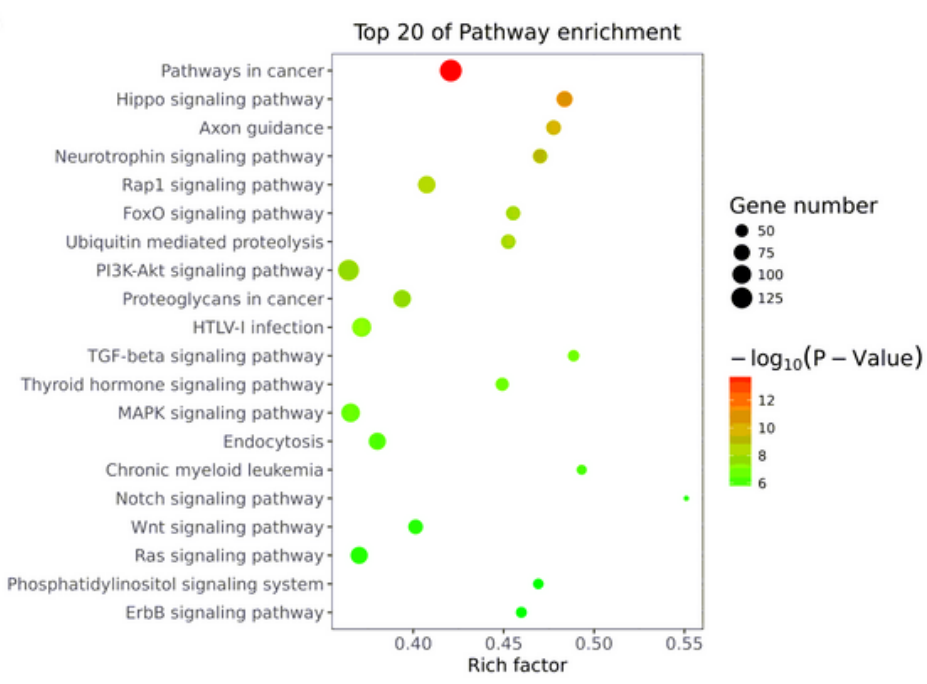

B

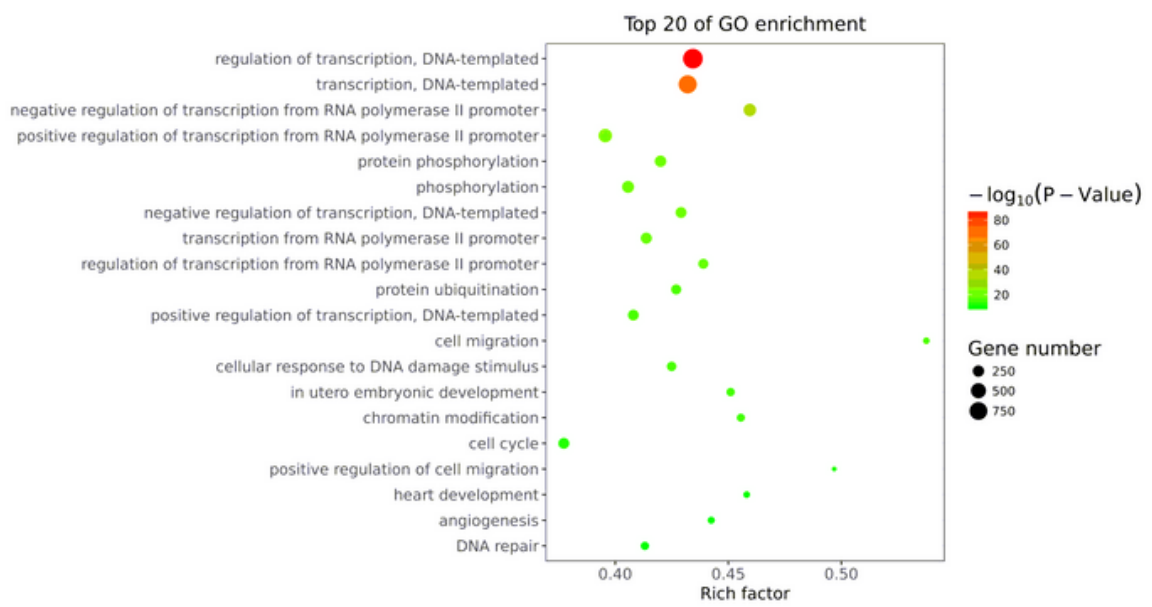

C

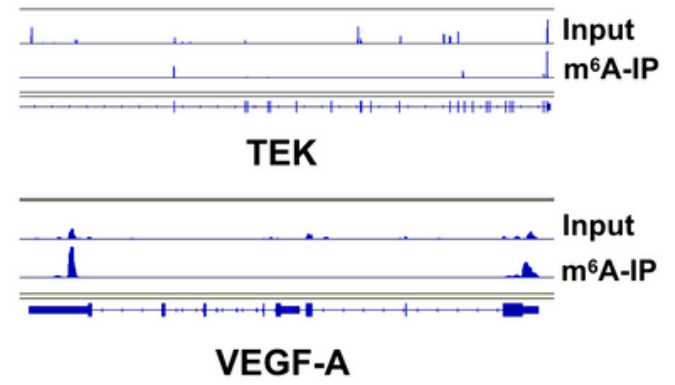

D

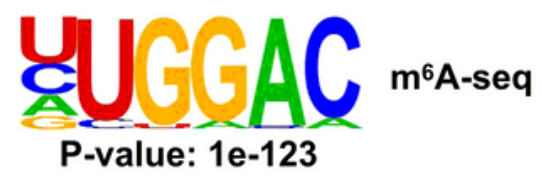

\section{Figure 5}

Mett|3-mediated m6A modification of genes correlated with PI3K pathway and angiogenesis A. Top 20 of pathway enrichment related to regulation by Mettl3 was displayed by bubble chart. B. The m6A-enriched genes were analyzed by Gene Ontology Biological Process exhibiting top $20 \mathrm{GO}$ enrichment process. C. IGV tracks showing the distributions of m6A reads across TEK and VEGF-A mRNAs generated from m6A- 
seq. D. The most common methylated consensus motif of 'GGAC' catalyzed by Mettl3 was determined by HOMER (http://homer.ucsd.edu/homer/ngs/peakMotifs.html).

A

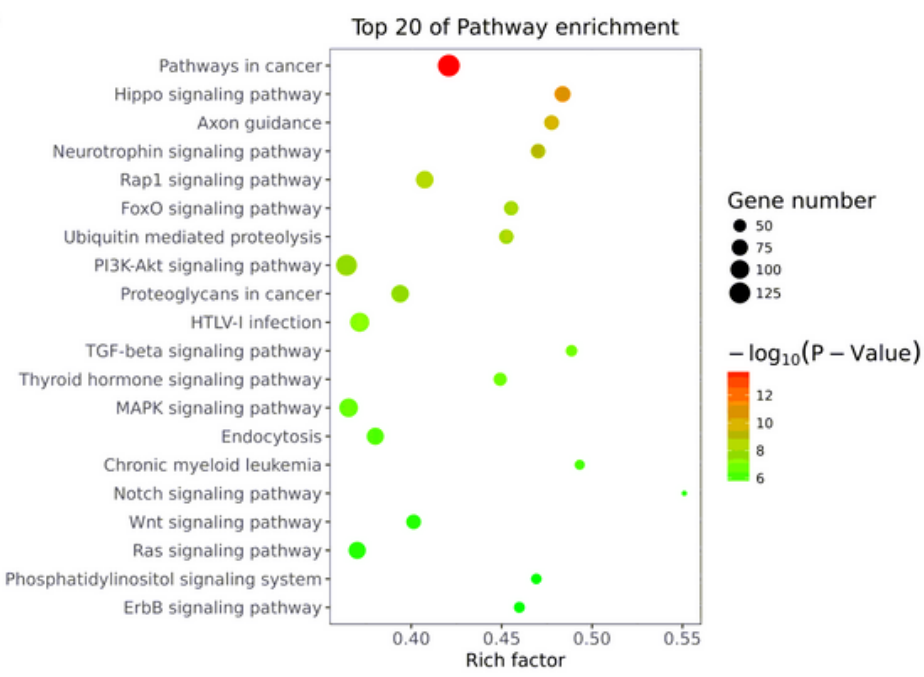

B

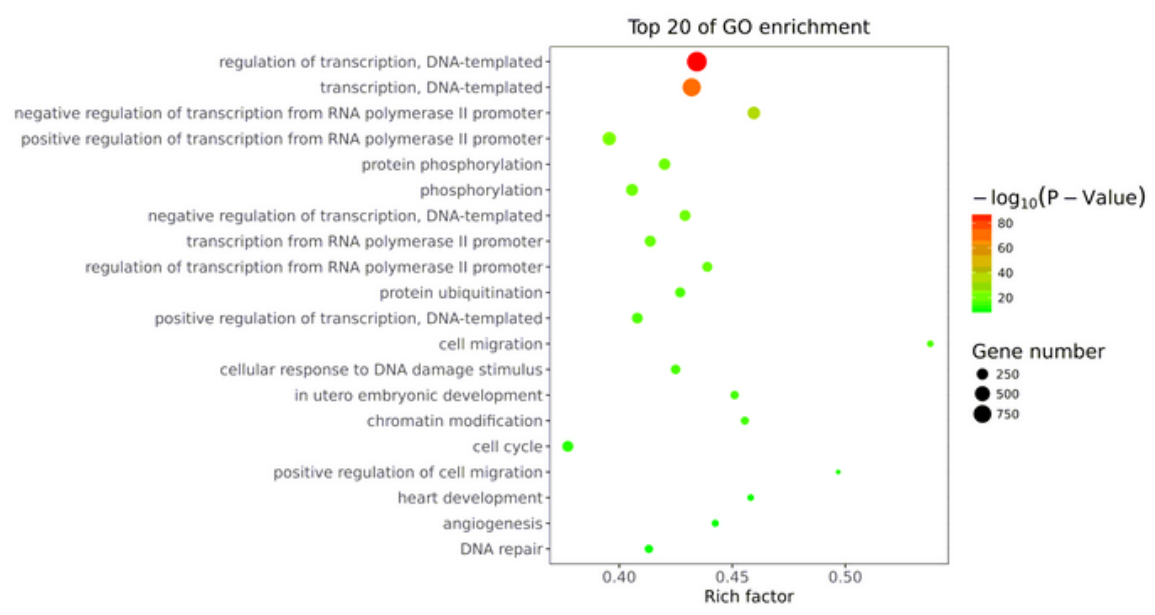

C
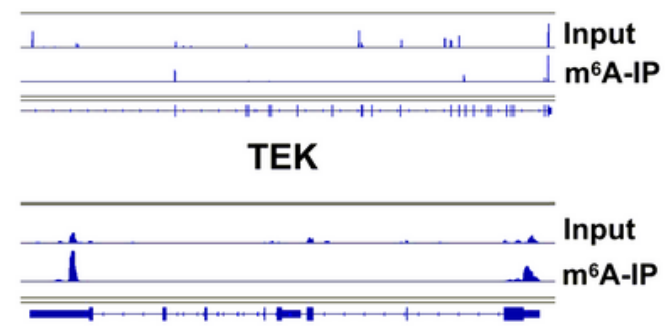

VEGF-A

D

\section{U⿺辶⿸丆巳一丶}

\section{Figure 5}

Mettl3-mediated m6A modification of genes correlated with PI3K pathway and angiogenesis A. Top 20 of pathway enrichment related to regulation by Mettl3 was displayed by bubble chart. B. The m6A-enriched genes were analyzed by Gene Ontology Biological Process exhibiting top $20 \mathrm{GO}$ enrichment process. C. 
IGV tracks showing the distributions of m6A reads across TEK and VEGF-A mRNAs generated from m6Aseq. D. The most common methylated consensus motif of 'GGAC' catalyzed by Mettl3 was determined by HOMER (http://homer.ucsd.edu/homer/ngs/peakMotifs.html).

A

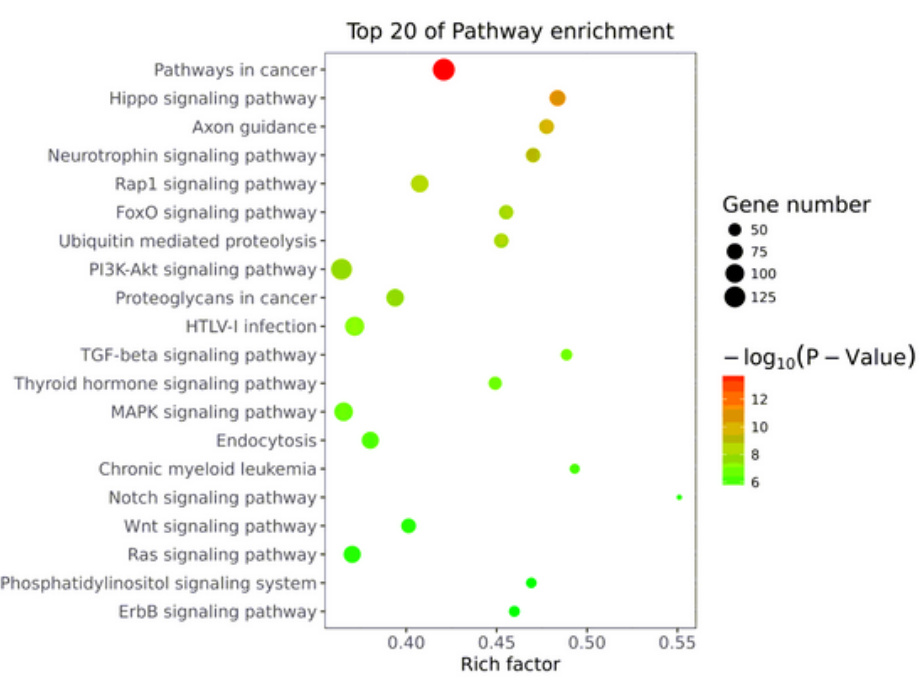

\section{B}

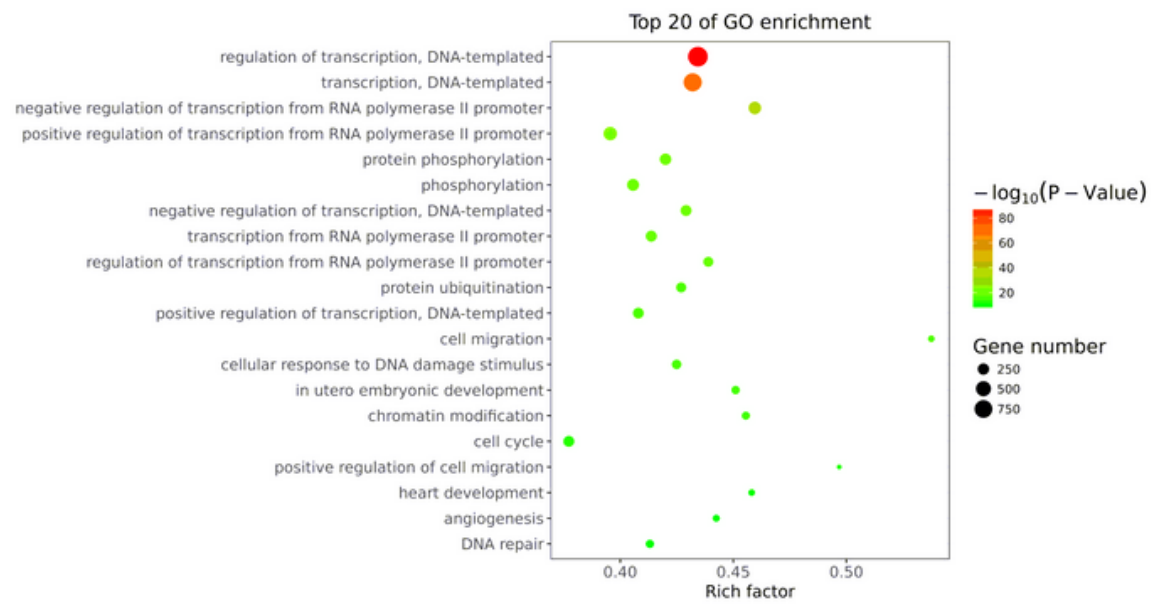

C
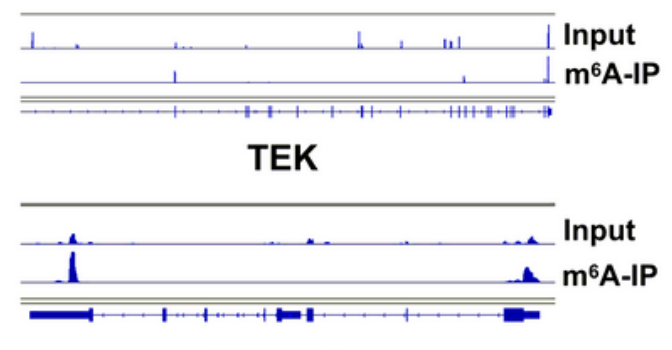

VEGF-A
D

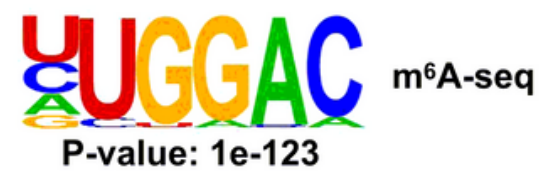

P-value: 1e-123

\section{Figure 5}

Mettl3-mediated m6A modification of genes correlated with PI3K pathway and angiogenesis A. Top 20 of pathway enrichment related to regulation by Mettl3 was displayed by bubble chart. B. The m6A-enriched 
genes were analyzed by Gene Ontology Biological Process exhibiting top $20 \mathrm{GO}$ enrichment process. C. IGV tracks showing the distributions of m6A reads across TEK and VEGF-A mRNAs generated from m6Aseq. D. The most common methylated consensus motif of 'GGAC' catalyzed by Mettl3 was determined by HOMER (http://homer.ucsd.edu/homer/ngs/peakMotifs.html).

A

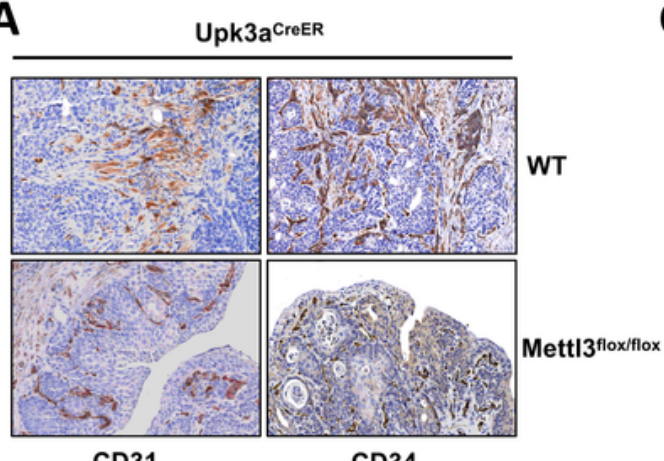

CD31

B
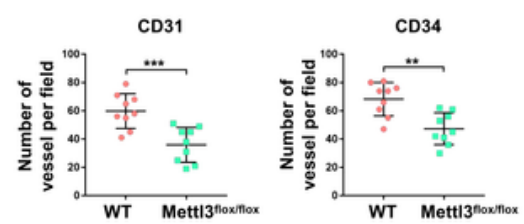

$\mathbf{E}$

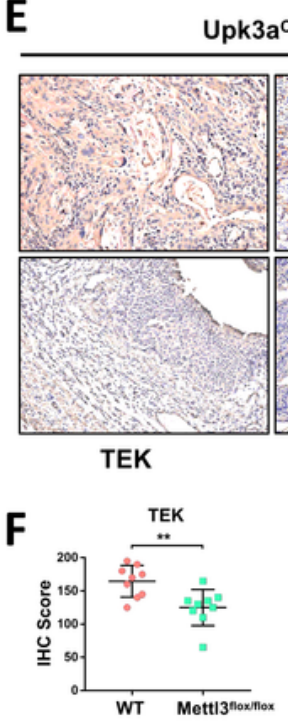

pk3aCreER

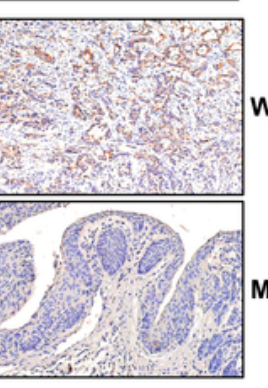

VEGF-A

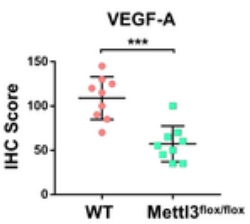

I

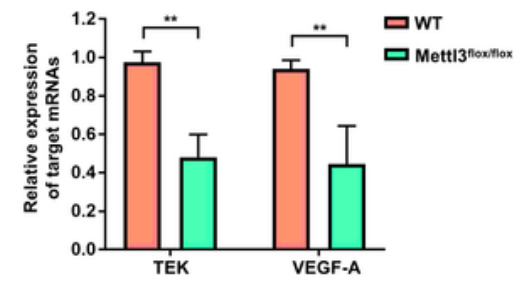

C

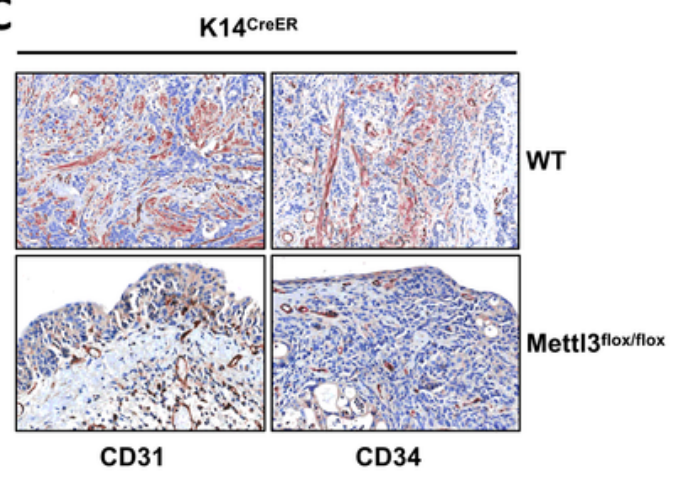

D

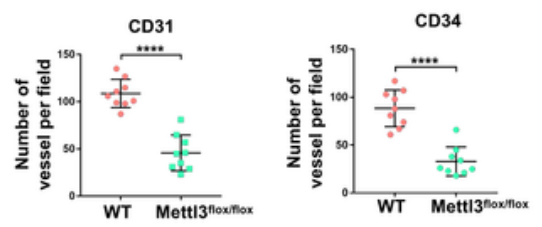

G

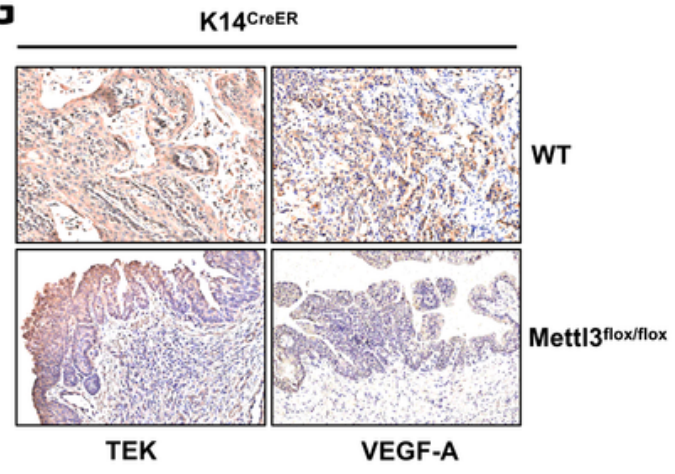

H
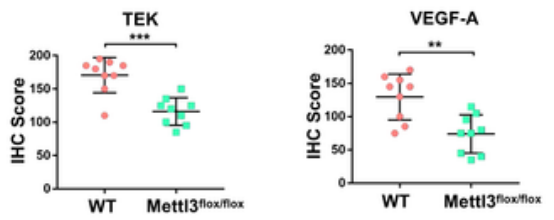

J

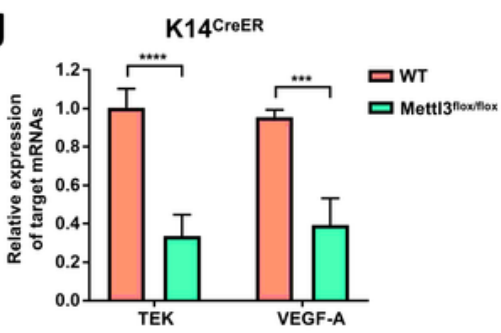

Figure 6 
Deletion of Mettl3 repressed TEK/VEGF-A-dependent tumor angiogenesis A. CD31 and CD34 were evaluated by ICH staining in Upk3aCreER WT (up) and Upk3aCreER Mettl3 KI (bottom) mice. Scale bars $100 \mu \mathrm{m}$. B. The expression level of CD31 (left) and CD34 (right) was counted on the basis of their staining intensity and positive cells under a double-blind assessment. C. Representative images showing IHC staining of CD31 (left) or CD34 (right) upon the sections obtained from K14CreER wild type and K14CreER Mett|3 KI mice. Scale bars $100 \mu \mathrm{m}$. D. The variation of CD31 or CD34 caused by deletion of Mettl3 among K14-oringinated cancer cells was quantified by IHC scoring. E. Representative images of ICH staining for Mettl3 targets. TEK and VEGF-A were dramatically reduced after specific ablation of Mettl3 in urothelial cells. Scale bars $100 \mu \mathrm{m}$. F. IHC score showing the difference of TEK and VEGF-A expression in tumor samples. G. Reduction of TEK and VEGF-A expression level by loss of Mettl3 in cells from which basal layer of the epithelium originated. Scale bars $100 \mu \mathrm{m}$. H. IHC score was computed according to the IHC staining of K14CreER mice with or without eliminating Mettl3. I. Examination of TEK and VEGF-A mRNAs level from bladder tissue with or without Mettl3 knockout by the qPCR experiments. $J$. The transcription level of TEK and VEGF-A in K14CreER mice was detected by qPCR. 
A

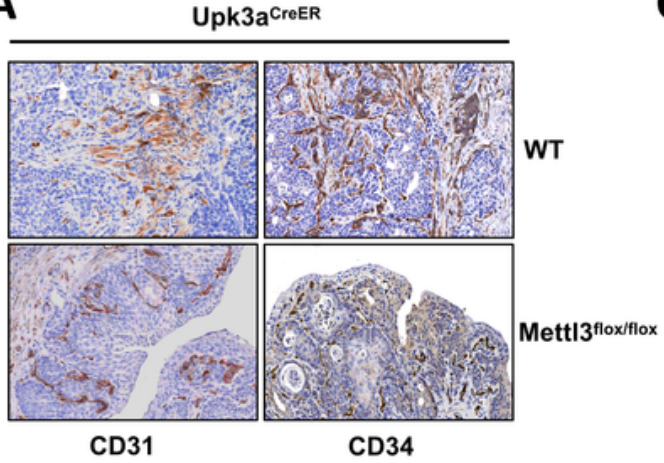

B

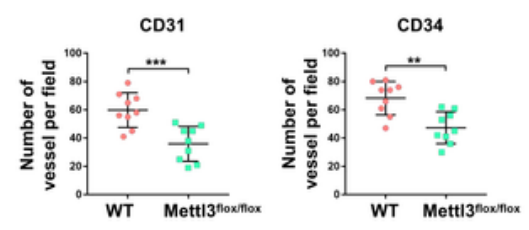

$\mathbf{E}$

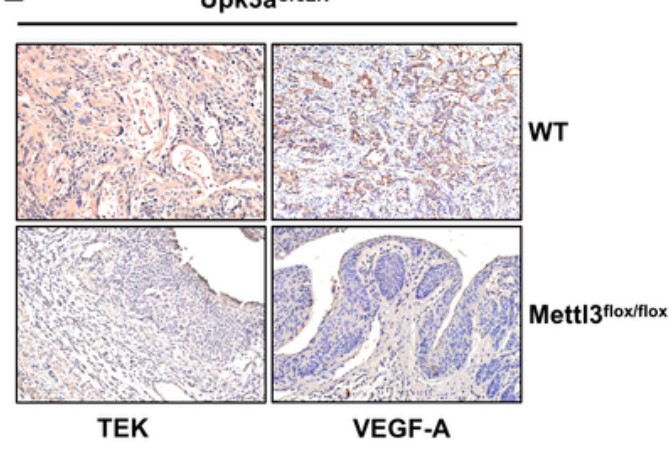

VEGF-A

C

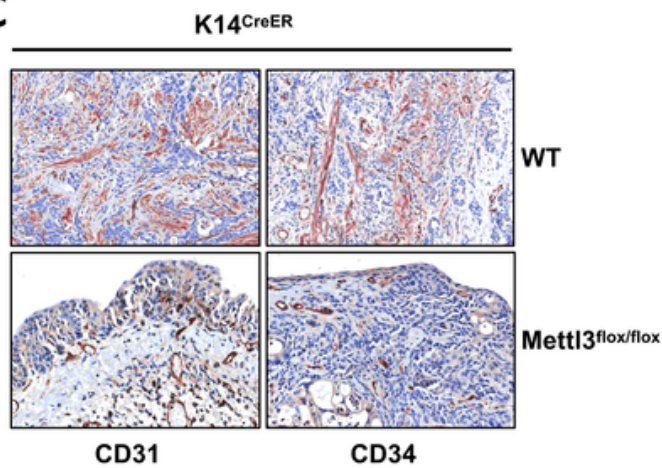

D

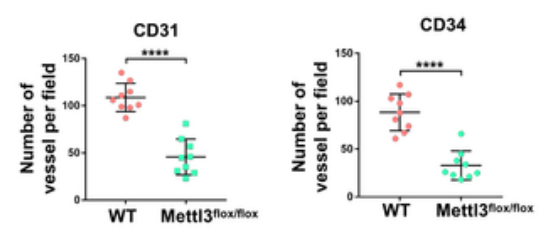

G

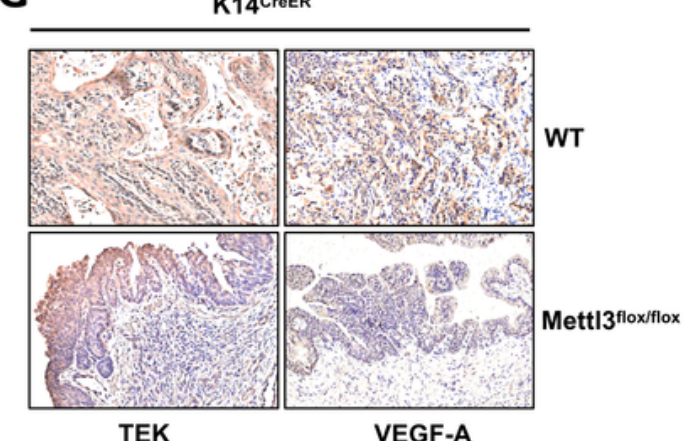

H

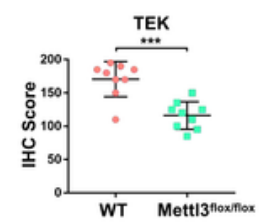

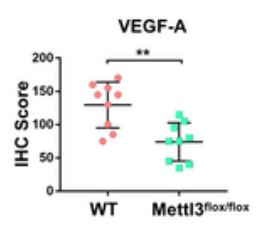

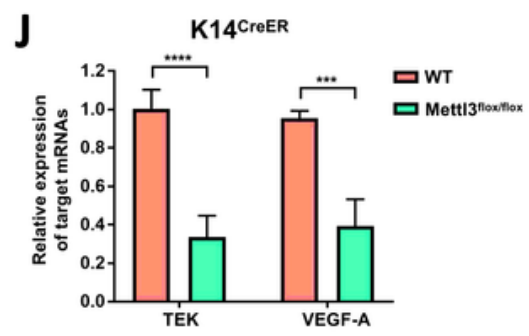

\section{Figure 6}

Deletion of Mettl3 repressed TEK/VEGF-A-dependent tumor angiogenesis A. CD31 and CD34 were evaluated by ICH staining in Upk3aCreER WT (up) and Upk3aCreER Mettl3 KI (bottom) mice. Scale bars $100 \mu \mathrm{m}$. B. The expression level of CD31 (left) and CD34 (right) was counted on the basis of their staining intensity and positive cells under a double-blind assessment. C. Representative images showing IHC staining of CD31 (left) or CD34 (right) upon the sections obtained from K14CreER wild type and 
K14CreER Mettl3 KI mice. Scale bars $100 \mu \mathrm{m}$. D. The variation of CD31 or CD34 caused by deletion of Mettl3 among K14-oringinated cancer cells was quantified by IHC scoring. E. Representative images of ICH staining for Mettl3 targets. TEK and VEGF-A were dramatically reduced after specific ablation of Mettl3 in urothelial cells. Scale bars $100 \mu \mathrm{m}$. F. IHC score showing the difference of TEK and VEGF-A expression in tumor samples. G. Reduction of TEK and VEGF-A expression level by loss of Mettl3 in cells from which basal layer of the epithelium originated. Scale bars $100 \mu \mathrm{m}$. H. IHC score was computed according to the IHC staining of K14CreER mice with or without eliminating Mettl3. I. Examination of TEK and VEGF-A mRNAs level from bladder tissue with or without Mettl3 knockout by the qPCR experiments. J. The transcription level of TEK and VEGF-A in K14CreER mice was detected by qPCR. 
A

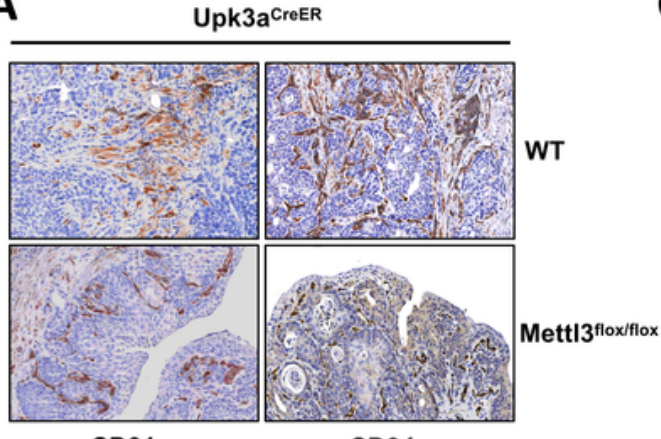

CD31

CD34
B

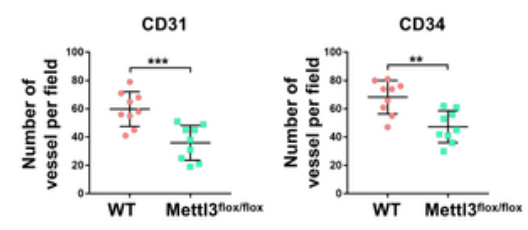

C

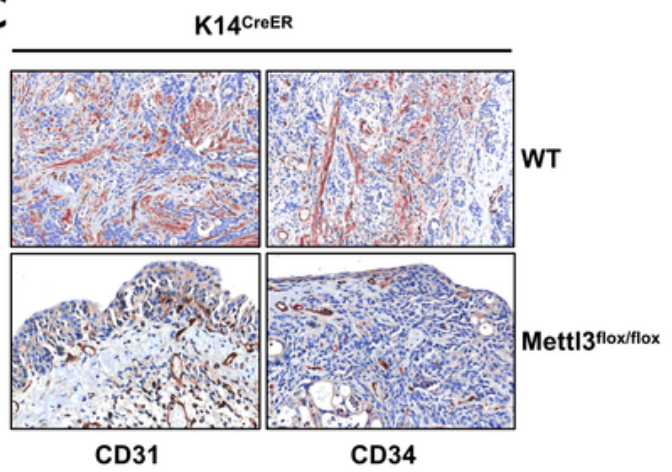

D

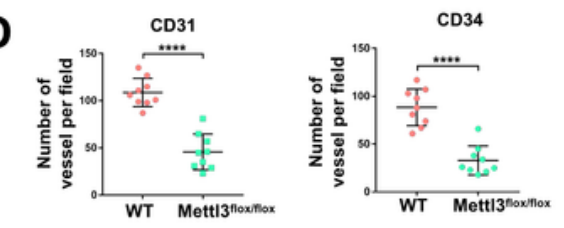

G

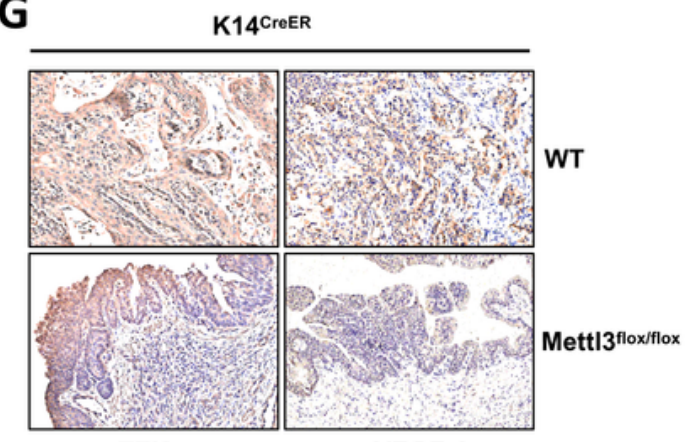

TEK

VEGF-A

H
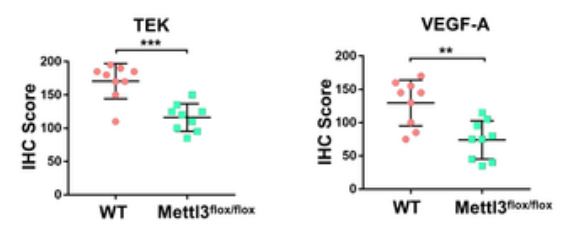

J

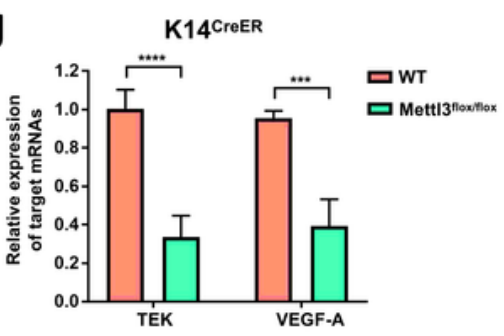

\section{Figure 6}

Deletion of Mettl3 repressed TEK/VEGF-A-dependent tumor angiogenesis A. CD31 and CD34 were evaluated by ICH staining in Upk3aCreER WT (up) and Upk3aCreER Mettl3 KI (bottom) mice. Scale bars $100 \mu \mathrm{m}$. B. The expression level of CD31 (left) and CD34 (right) was counted on the basis of their staining intensity and positive cells under a double-blind assessment. C. Representative images showing IHC staining of CD31 (left) or CD34 (right) upon the sections obtained from K14CreER wild type and 
K14CreER Mettl3 KI mice. Scale bars $100 \mu \mathrm{m}$. D. The variation of CD31 or CD34 caused by deletion of Mettl3 among K14-oringinated cancer cells was quantified by IHC scoring. E. Representative images of ICH staining for Mettl3 targets. TEK and VEGF-A were dramatically reduced after specific ablation of Mettl3 in urothelial cells. Scale bars $100 \mu \mathrm{m}$. F. IHC score showing the difference of TEK and VEGF-A expression in tumor samples. G. Reduction of TEK and VEGF-A expression level by loss of Mettl3 in cells from which basal layer of the epithelium originated. Scale bars $100 \mu \mathrm{m}$. H. IHC score was computed according to the IHC staining of K14CreER mice with or without eliminating Mettl3. I. Examination of TEK and VEGF-A mRNAs level from bladder tissue with or without Mettl3 knockout by the qPCR experiments. J. The transcription level of TEK and VEGF-A in K14CreER mice was detected by qPCR. 